\title{
A Simplified Finite Difference Method (SFDM) for EMHD Powell-Eyring Nanofluid Flow Featuring Variable Thickness Surface and Variable Fluid Characteristics
}

\author{
M. Irfan, ${ }^{1}$ M. Asif Farooq, ${ }^{1}$ T. Iqra, ${ }^{1}$ A. Mushtaq $\mathbb{D}^{2},{ }^{2}$ and Z. H. Shamsi $\mathbb{D}^{3}$ \\ ${ }^{1}$ Department of Mathematics, School of Natural Sciences (SNS), National University of Sciences and Technology (NUST), \\ Sector H-12, Islamabad 44000, Pakistan \\ ${ }^{2}$ Seksjon for Matematikk, Nord Universitet, Bodø 8026, Norway \\ ${ }^{3}$ Department of Mathematics, University of the Punjab, Lahore 54590, Pakistan
}

Correspondence should be addressed to A. Mushtaq; asif.mushtaq@nord.no

Received 26 August 2020; Revised 30 September 2020; Accepted 9 October 2020; Published 26 November 2020

Academic Editor: Taha Aziz

Copyright $\odot 2020$ M. Irfan et al. This is an open access article distributed under the Creative Commons Attribution License, which permits unrestricted use, distribution, and reproduction in any medium, provided the original work is properly cited.

\begin{abstract}
We study constant and variable fluid properties together to investigate their effect on MHD Powell-Eyring nanofluid flow with thermal radiation and heat generation over a variable thickness sheet. The similarity variables assist in having ordinary differential equations acquired from partial differential equations (PDEs). A novel numerical procedure, the simplified finite difference method (SFDM), is developed to calculate the physical solution. The SFDM described here is simple, efficient, and accurate. To highlight its accuracy, results of the SFDM are compared with the literature. The results obtained from the SFDM are compared with the published results from the literature. This gives a good agreed solution with each other. The velocity, temperature, and concentration distributions, when drawn at the same time for constant and variable physical features, are observed to be affected against incremental values of the flow variables. Furthermore, the impact of contributing flow variables on the skin friction coefficient (drag on the wall) and local Nusselt (heat transfer rate on the wall) and Sherwood numbers (mass transfer on the wall) is illustrated by data distributed in tables. The nondimensional skin friction coefficient experiences higher values for constant flow regimes especially in comparison with changing flow features.
\end{abstract}

\section{Introduction}

Nowadays, the use of thermal analysis in industry for nonNewtonian fluids is undergoing far reaching consequences covering the processes in biology to the mechanical devices, namely, electronics machinery. Therefore, it is worth investigating to optimize the flow of heat transfer for the system. One way in which the underlying system's thermal conductivity is enhanced is to use the nanofluids. The concept of variable thickness surface is helpful in reducing the weight of structural elements.

Magnetohydrodynamics (MHD) deals with the interaction between electrically conducting fluid and magnetic field. Studying MHD flow has great value towards learning metallurgical and metal-working processes.

A nanofluid is a mixture of nanometer sized particle and base fluid. Nanofluids provide numerous uses in manufacturing, electronics, physical science, and biotechnology. The nanofluids demonstrate improved thermal conductivity due to their unique physical properties. Therefore, considering nanofluids is one of the ways in enhancing thermal conductivity.

Daniel et al. [1] showed that the nanofluid flow over a variable sheet thickness is sensitive to a thermal radiation with an increase in temperature. Fang et al. [2] provided the 
flow results adjacent to variable thicked surface. Alsaedi et al. [4] provided analysis for the MHD bioconvective nanofluid with gyrotactic microorganisms. A similar study by Atif et al. [3] discussed micropolar nanofluid. The magnetic effect on a third-grade fluid with chemically reactive species has been put forward in [5]. In their study, Khan et al. [6] discussed stagnation point flow with variable properties and obtained solution numerically. Hayat et al. [7] studied stagnation point flow for carbon-water nanofluid. The studies lead by Yasmeen et al. [8] and Hayat et al. [10] have shown flow over a sheet. In the study of Yasmeen et al. [8], the authors assumed ferrorfluid in their study, but Hayat et al. [10] considered melting heat transfer effects. In their work, Hayat et al. $[9,11]$ considered variable properties, but in the latter work, they discussed unsteady three dimensional convection flows. Das et al. [12] underlined analysis on second-grade MHD fluid flows. Bhatacharyya et al. [13] set up exact solution with varying wall temperature for Casson fluid. Raju et al. [14] used FEM to find the solution of unsteady MHD convection Couette flow. Farooq et al. [15] reported fluid flow adjacent to a variable thicked Riga plate. Kumar et al. [16] discussed Brownian motion, slip effects, and thermophoresis effects near a variable thicked surface. Anantha et al. [17] described bioconvective fluid flow in Carreau fluid adjacent to variable thicked surface. Kumar et al. [18] addressed MHD Cattaneo-Christov flow past a cone as well as wedge in their research. Daniel et al. [19] recorded MHD nanofluid flow with slip and convective conditions. Salahuddin et al. [20] used numerical method to present magnetic Williamson flow of the Cattaneo-Christov heat flux model with varying thickness. Reddy et al. [21] took MHD Williamson nanofluid flow with varying physical properties near a variable thickness sheet. Malik et al. [22] discussed variable viscosity and MHD flow for Casson fluid by using the Keller-Box method. The work on variable thickness and radiation for Casson fluid is presented in [23]. Hydromagnetic distribution in nanofluid flow with variable viscosity and radially stretching sheet has been discussed in [24]. In [25], they reported a solution of MHD flow of nanofluid with variable thickness. Turkyilmazoglu [26] mentioned effects of radiation with varied viscosity on a time-dependent MHD porous flow. Prasad et al. [27] noticed MHD fluid flow solution over a stretching surface with thermophysical varying features. Vajravelu et al. [28] discussed variable fluid flow characteristics over a vertical surface. Das [29], Mukhopadhyay [30], and Rahman and Eltayeb [31] reported the effect of variable properties in fluid flows. Hayat et al. [32] discussed melting heat transfer for Powell-Eyring fluid. Jalil and Asghar [33] also used Powell-Eyring fluid for their analysis and found a solution by the Lie group method. Fluid flow with numerical and series solutions over an exponentially stretchable surface with the Powell-Eyring model has been discussed in [34]. In [35], the flow of Powell-Eyring non-Newtonian fluid has been discussed. Mustafa et al. [36] discussed MHD boundary layer nanofluid for second-grade fluid. Motsumi et al. [37] discussed thermal radiation and viscous dissipation on boundary layer flow of nanofluids over a permeable moving flat plate. In their work, Andersson and Aarseth [39] revisited Sakiadis flow with variable fluid properties. For nanofluid and other relevant areas of research, it would be worth reading these references [40-44].

Constant fluid properties of Newtonian and non-Newtonian fluids have been found in most of the above studies. Variable fluid property analysis is uncommon with variable sheet thickness. The current work is an extension of the work presented in Irfan et al. [45] where the Newtonian nanofluid is considered. To the best of the authors' knowledge, no research has been done on the Powell-Eyring nanofluid with constant and variable fluid properties together. To fill this gap, under the influence of different kinematics and dynamics, we examine constant and variable fluid properties to find critical difference between these when the non-Newtonian Powell-Eyring fluid is taken into account. Furthermore, we introduce a new numerical procedure, SFDM, to find the solution of the ordinary differential equations (ODEs).

The novelty of the current work lies in addressing the nanofluid of Powell-Eyring along with constant and variable fluid properties. A novel computational technique, the SFDM, has been tested for the Powell-Eyring nanofluids in addition to theoretical modelling of fluid flow. The SFDM is simple, accurate, and easy to implement in MATLAB.

The layout of the paper is as follows. Section 2 is devoted to obtaining mathematical equations of the physical system. Section 3 deals with both constant and variable fluid features. Section 4 offers overview of a numerical process. Section 5 describes the results and the discussion. The findings of the paper are summed up in Section 6.

\section{Theoretical Model}

Consider a magnetohydrodynamic (MHD) two-dimensional steady laminar flow of Powell-Eyring nanofluid over a nonlinear uneven stretching sheet emerging from the narrow slit with variable fluid characteristics. Assume that varying magnetic field is directed perpendicular to the flow motion and is defined by $B_{1}\left(x_{1}\right)=B_{0}\left(x_{1}+b\right)^{n-1 / 2}$. In addition, variable electric field is chosen as $E_{1}^{*}\left(x_{1}\right)=E_{0}\left(x_{1}+b\right)^{n-1 / 2}$. Also, $K\left(x_{1}\right)=K_{0}\left(x_{1}+b\right)^{1-n}$ is the variable permeability. The surface has a nonlinear stretching velocity $U_{w}=a_{0}\left(x_{1}+b\right)^{n}$. Furthermore, the thickness of the sheet is varied by the relation $y_{1}=A\left(x_{1}+b\right)^{1-n / 2}$, in which $A$ is a very small constant. Fluid flow configuration is illustrated in Figure 1. The assumption of small magnetic Reynolds number leads to disregarding the induced magnetic field.

After incorporating aforementioned fluid flow assumptions in the following equations of motion, we get [45] 


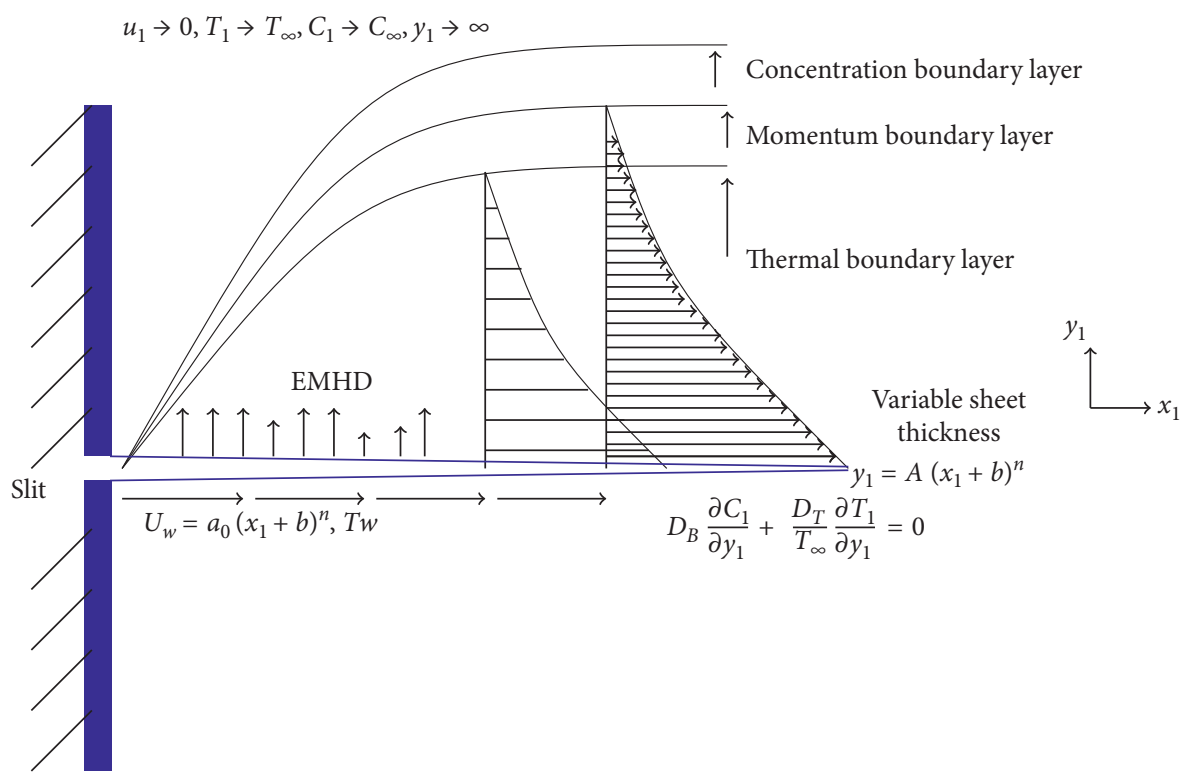

Figure 1: Geometry of the problem.

$$
\begin{aligned}
\frac{\partial u_{1}}{\partial x_{1}}+\frac{\partial v_{1}}{\partial y_{1}}= & 0 \\
u_{1} \frac{\partial u_{1}}{\partial x_{1}}+v_{1} \frac{\partial u_{1}}{\partial y_{1}}= & \frac{1}{\rho_{1}} \frac{\partial}{\partial y_{1}}\left(\mu_{1}\left(T_{1}\right) \frac{\partial u_{1}}{\partial y_{1}}\right)+\frac{1}{\rho_{1} \beta_{1} d} \frac{\partial^{2} u_{1}}{\partial y_{1}^{2}}-\frac{1}{2 \rho_{1} \beta_{1} d^{3}}\left(\frac{\partial u_{1}}{\partial y_{1}}\right)^{2} \frac{\partial^{2} u_{1}}{\partial y_{1}^{2}} \\
& +\frac{\sigma}{\rho_{1}}\left(E_{1}^{*}\left(x_{1}\right) B_{1}\left(x_{1}\right)-B_{1}^{2}\left(x_{1}\right) u_{1}\right)+g \beta\left(T_{1}-T_{\infty}\right)-\frac{\mu_{1} u_{1}}{\rho_{1} K\left(x_{1}\right)} \\
u_{1} \frac{\partial T_{1}}{\partial x_{1}}+v_{1} \frac{\partial T_{1}}{\partial y_{1}=} & \frac{1}{\rho_{1} C_{p}} \frac{\partial}{\partial y_{1}}\left(k_{1}\left(T_{1}\right) \frac{\partial T_{1}}{\partial y_{1}}\right)-\frac{1}{\rho_{1} C_{p}} \frac{\partial q_{r}}{\partial y_{1}}+\frac{\sigma}{\rho_{1} C_{p}}\left(u_{1} B_{1}\left(x_{1}\right)-E_{1}^{*}\left(x_{1}\right)\right)^{2}+\frac{Q_{1}\left(x_{1}\right)}{\rho C_{p}}\left(T_{1}-T_{\infty}\right) \\
& +\tau\left(D_{B} \frac{\partial T_{1}}{\partial y_{1}} \frac{\partial C_{1}}{\partial y_{1}}+\frac{D_{T}}{T_{\infty}}\left(\frac{\partial T_{1}}{\partial y_{1}}\right)^{2}\right) \\
u_{1} \frac{\partial C_{1}}{\partial x_{1}}+v_{1} \frac{\partial C_{1}}{\partial y_{1}}= & D_{B} \frac{\partial^{2} C_{1}}{\partial y_{1}^{2}}+\frac{D_{T}}{T_{\infty}} \frac{\partial^{2} T_{1}}{\partial y_{1}^{2}},
\end{aligned}
$$

where $\left(u_{1}, v_{1}\right), \mu_{1}, \rho_{1}$, and $C_{p}$ are the velocity components, the dynamic viscosity, the density, and the specific heat capacity, respectively. Moreover, $E_{1}^{*}\left(x_{1}\right), B_{1}\left(x_{1}\right), T_{1}$, and $C_{1}$ are the electric field, the magnetic field, the temperature of the fluid, and nanoparticle volume fraction, respectively. Also, $T_{w}$ is the wall temperature, and the ambient temperature is denoted by $T_{\infty}$. The parameters $D_{B}$ and $D_{T}$ are characterized as the Brownian diffusion and thermophoretic diffusion coefficients, respectively. In $\tau=\left(\rho_{1} c\right)_{p} /\left(\rho_{1} c\right)_{f}$, $\left(\rho_{1} c\right)_{p}$ is the effective heat capacity of the nanoparticle and $\left(\rho_{1} c\right)_{f}$ is the heat capacity of the fluid. $q_{r}$ represents radiation due to heat flow, and $Q_{1}\left(x_{1}\right)$ is heat generation/absorption parameter.

The boundary conditions needed to solve (1)-(4) are given by

$$
\begin{aligned}
& u_{1}=U_{w}\left(x_{1}\right)=a_{o}\left(x_{1}+b\right)^{n}, v_{1}=0, T_{1}=T_{w}, D_{B} \frac{\partial C_{1}}{\partial y_{1}}+\frac{D_{T}}{T_{\infty}} \frac{\partial T_{1}}{\partial y_{1}}=0 \text { at } y_{1}=A\left(x_{1}+b\right)^{1-n / 2}, \\
& u_{1} \longrightarrow 0, T_{1} \longrightarrow T_{\infty}, C_{1} \longrightarrow C_{\infty} \text { as } y_{1} \longrightarrow \infty
\end{aligned}
$$


With the following transformations $[1,15]$ :

$$
\begin{aligned}
\xi & =\sqrt{\left(\frac{n+1}{2}\right) \frac{a_{0}\left(x_{1}+b\right)^{n-1}}{v_{0}}} y_{1}, \\
\psi & =\sqrt{\frac{2}{n+1} v_{0} a_{0}\left(x_{1}+b\right)^{n+1}} F(\xi), \\
\alpha= & A\left(\frac{(n+1) a_{0}}{2 v_{0}}\right)^{1 / 2}, \\
\eta= & \xi-\alpha=y_{1} \sqrt{\left(\frac{n+1}{2}\right) \frac{a_{0}\left(x_{1}+b\right)^{n-1}}{\nu_{0}}}-\alpha, \\
\widetilde{\Theta}= & \frac{T_{1}-T_{\infty}}{T_{w}-T_{\infty}}, \\
\widetilde{\Phi}= & \frac{C_{1}-C_{\infty}}{C_{w}-C_{\infty}}, \\
u_{1}= & a_{0}\left(x_{1}+b\right)^{n} \widetilde{F}^{\prime}(\xi), \\
v_{1}= & -\sqrt{\frac{n+1}{2} v_{0} a_{0}\left(x_{1}+b\right)^{n-1}} \widetilde{F}(\xi) \\
& -\xi\left(\frac{n-1}{n+1}\right) \widetilde{F}^{\prime}(\xi) \sqrt{\frac{n+1}{2} v_{0} a_{0}\left(x_{1}+b\right)^{n-1}},
\end{aligned}
$$

equation (1) is satisfied through $\psi$. In the above, $v_{0}=\mu_{0} / \rho_{1}$ is the ambient kinematic viscosity. By using above transformations, equations (2)-(4) resulted into

$$
\begin{aligned}
& \left(\frac{\mu_{1}}{\mu_{o}} \widetilde{F}^{\prime \prime}\right)^{\prime}+N \widetilde{F}^{\prime \prime \prime}-N \lambda\left(\frac{n+1}{2}\right) \widetilde{F}^{\prime^{2}} \widetilde{F}^{\prime \prime \prime}-\frac{2 n}{n+1} \widetilde{F}^{\prime^{2}} \\
& +\widetilde{F} \widetilde{F}^{\prime \prime}+M\left(E-\widetilde{F} \mathrm{X}^{\prime}\right)-\mathrm{Kp} \frac{\mu_{1}}{\mu_{o}} \widetilde{F}^{\prime}+\mathrm{Gr} \widetilde{\Theta}=0, \\
& \left(1+\frac{4}{3} \mathrm{Rd}\right)\left(\frac{k_{1}}{k_{0}} \widetilde{\Theta}^{\prime}\right)^{\prime}+\operatorname{Pr}_{0}\left(\widetilde{F} \Theta^{\prime}+\mathrm{Nb} \widetilde{\Theta}^{\prime} \widetilde{\Phi}^{\prime}+\mathrm{Nt}\left(\widetilde{\Theta}^{\prime}\right)^{2}\right. \\
& \left.\quad+\mathrm{MEc}\left(\widetilde{F}^{\prime}-E\right)^{2}+\frac{2}{n+1} s \widetilde{\Theta}\right)=0, \\
& \widetilde{\Phi}^{\prime \prime}+\frac{\mathrm{Nt}}{\mathrm{Nb}} \widetilde{\Theta}^{\prime \prime}+\operatorname{LePr}_{0} \widetilde{F} \widetilde{\Phi}^{\prime}=0 .
\end{aligned}
$$

The transformed boundary conditions are written as

$$
\begin{aligned}
& \widetilde{F}=\alpha\left(\frac{1-n}{1+n}\right), \widetilde{F}^{\prime}=1, \operatorname{Nb} \widetilde{\Phi}^{\prime}+N t \widetilde{\Theta}^{\prime}=0, \widetilde{\Theta}=1, \text { at } \\
& \alpha=A\left(\frac{(n+1) a_{0}}{2 v_{0}}\right)^{1 / 2} \widetilde{\Theta}=0, \widetilde{F}^{\prime}=0, \widetilde{\Phi}=0 \text {, when } \xi \longrightarrow \infty .
\end{aligned}
$$

Assuming $\widetilde{F}(\xi)=\widetilde{f}(\xi-\alpha)=\widetilde{f}(\eta)$, we get

$$
\begin{aligned}
& \left(\frac{\mu_{1}}{\mu_{0}} \tilde{f}^{\prime \prime}\right)^{\prime}+N \tilde{f}^{\prime \prime \prime}-N \lambda\left(\frac{n+1}{2}\right) \tilde{f}^{\prime \prime} \tilde{f}^{\prime \prime \prime}-\frac{2 n}{n+1} \tilde{f}^{\prime} 2+\tilde{f} \tilde{f}^{\prime \prime} \\
& +M\left(E-\tilde{f}^{\prime}\right)-\operatorname{Kp} \frac{\mu_{1}}{\mu_{o}} \widetilde{f}^{\prime}+\mathrm{Gr} \tilde{\theta}=0 \\
& \left(1+\frac{4}{3} \operatorname{Rd}\right)\left(\frac{k_{1}}{k_{0}} \widetilde{\theta}^{\prime}\right)^{\prime}+\operatorname{Pr}_{0}\left(\tilde{f} \theta^{\prime}+\mathrm{Nb} \widetilde{\theta}^{\prime} \widetilde{\phi}^{\prime}+\operatorname{Nt}\left(\widetilde{\theta}^{\prime}\right)^{2}\right. \\
& \left.+\operatorname{MEc}\left(\tilde{f}^{\prime}-E\right)^{2}+\frac{2}{n+1} s \tilde{\theta}\right)=0 \\
& \widetilde{\phi}^{\prime \prime}+\frac{\mathrm{Nt}}{\mathrm{Nb}} \tilde{\theta}^{\prime \prime}+\operatorname{LePr}_{0} \tilde{f} \widetilde{\phi}^{\prime}=0
\end{aligned}
$$

The transformed boundary conditions on the new domain are written as

$$
\begin{aligned}
& \widetilde{f}=\alpha\left(\frac{1-n}{1+n}\right), \widetilde{f}^{\prime}=1, \mathrm{Nb} \widetilde{\phi}^{\prime}+\mathrm{Nt} \widetilde{\theta}^{\prime}=0, \widetilde{\theta}=1, \text { at } \\
& \eta=0, \widetilde{\theta}=0, \widetilde{f}^{\prime}=0, \widetilde{\phi}=0, \text { when } \eta \longrightarrow \infty .
\end{aligned}
$$

The quantities appearing above are grouped into

$$
\begin{aligned}
& M=\frac{2 \sigma B_{o}^{2}}{\rho a_{0}(n+1)}, \\
& E=\frac{E_{0}}{B_{0} a_{0}\left(x_{1}+b\right)^{n}}, \\
& \mathrm{Kp}=\frac{2 v_{o}}{K_{o} a_{0}(n+1)}, \\
& N=\frac{1}{d \beta_{1} \mu_{o}}, \\
& \lambda=\frac{a_{0}^{3}\left(x_{1}+b\right)^{3 n-1}}{2 d^{2} v_{0}} \\
& \operatorname{Pr}_{0}=\frac{\mu_{0} C_{p}}{k_{0}}, \\
& \mathrm{Nb}=\frac{\tau D_{B}\left(C_{w}-C_{\infty}\right)}{v_{0}}, \\
& \mathrm{Nt}=\frac{\tau D_{T}\left(T_{w}-T_{\infty}\right)}{T_{\infty} \nu_{0}}, \\
& E_{c}=\frac{U_{w}^{2}}{C_{p}\left(T_{w}-T_{\infty}\right)}, \\
& \mathrm{Rd}=\frac{4 \sigma T_{\infty}^{3}}{k_{o} k^{*}},
\end{aligned}
$$




$$
\begin{aligned}
s & =\frac{Q_{o}}{\rho a_{0} C_{p}}, \\
\mathrm{Gr} & =\frac{2 g \beta\left(T_{w}-T_{\infty}\right)}{a_{0}^{2}(n+1)(x+b)^{2 n-1}}, \\
\mathrm{Le} & =\frac{v_{0}}{D_{B}},
\end{aligned}
$$

where $\alpha$ is the wall thickness parameter, $b$ is a positive constant, $a_{0}$ is a rate of stretching sheet, Ec is the Eckert number, $\epsilon$ is the thermal conductivity parameter, $M$ is a magnetic parameter, $k_{1}$ is the thermal conductivity of the fluid, $s$ is a heat source parameter, $\sigma$ is the electrical conductivity, $\sigma^{*}$ is the Stefan-Boltzmann constant, $\operatorname{Pr}_{0}$ is the ambient Prandtl number, Le is the Lewis number, $\mathrm{Nb}$ is the Brownian parameter, $\mathrm{Nt}$ is the thermophoresis parameter, $\lambda$ is a free stream parameter, $\mathrm{Rd}$ is a thermal radiation parameter, $\mathrm{Kp}$ is a permeability parameter, $k^{*}$ is the mean absorption coefficient, $\mathrm{Gr}$ is the Grashof number, and $B_{0}$ is applied magnetic field.

\section{Analysis on Fluid Properties}

First consider constant thermophysical properties of liquids followed by the variable physical properties.

3.1. Case A: Constant Fluid Features. In such scenario, equations (10)-(12) reduce into the following:

$$
\begin{aligned}
& \widetilde{f}^{\prime \prime \prime}+N \widetilde{f}^{\prime \prime \prime}-N \lambda\left(\frac{n+1}{2}\right) \tilde{f}^{\prime \prime} \tilde{f}^{\prime \prime \prime}-\frac{2 n}{n+1} \widetilde{f}^{{ }^{2}}+\widetilde{f} \widetilde{f}^{\prime \prime} \\
& +M\left(E-\tilde{f}^{\prime}\right)-K_{p} \tilde{f}^{\prime}+\operatorname{Gr} \tilde{\theta}=0, \\
& \left(1+\frac{4}{3} \mathrm{Rd}\right) \tilde{\theta}^{\prime \prime}+\operatorname{Pr}_{o}\left(\tilde{f} \widetilde{\theta}^{\prime}+\operatorname{Nb} \tilde{\theta}^{\prime} \widetilde{\phi}^{\prime}+\operatorname{Nt}\left(\tilde{\theta}^{\prime}\right)^{2}\right. \\
& \left.+\operatorname{MEc}\left(\tilde{f}^{\prime}-E\right)^{2}+\frac{2}{n+1} s \tilde{\theta}\right)=0 \\
& \widetilde{\phi}^{\prime \prime}+\frac{\mathrm{Nt}}{\mathrm{Nb}} \widetilde{\theta}^{\prime \prime}+\operatorname{Le} \tilde{f} \widetilde{\phi}^{\prime}=0
\end{aligned}
$$

Illustration of the skin friction coefficient in Table 1 reveals an outstanding alignment of the SFDM (discussed below) with Daniel et al. [1] and Fang et al. [2].

3.2. Case B: Variable Fluid Features. The variation in viscosity for water due to change in temperature is illustrated in Table 2 (see White [38]). The viscosity decreases by a factor of 6 . However, less change is noted in density. This motivates us to study the variable fluid properties. Therefore, viscosity and thermal conductivity vary accordingly with temperature [39].

$$
\mu_{1}(T)=\frac{\mu_{r}}{1+\gamma\left(T_{1}-T_{r}\right)}
$$

TABle 1: Presenting $-\tilde{f} \prime \prime(0)$ both for varying $n$ and specific $\alpha=$ 0.25 (Case A).

\begin{tabular}{lccc}
\hline$n$ & Fang et al. [2] & Daniel et al. [1] & Present result (SFDM) \\
\hline 10 & 1.1433 & 1.143316 & 1.143301 \\
9 & 1.1404 & 1.140388 & 1.140431 \\
7 & 1.1323 & 1.132281 & 1.132301 \\
5 & 1.1186 & 1.118587 & 1.118602 \\
3 & 1.0905 & 1.090490 & 1.090400 \\
0.5 & 0.9338 & 0.933828 & 0.933796 \\
$-1 / 3$ & 0.5000 & 0.500000 & 0.502557 \\
-0.5 & 0.0833 & 0.083289 & 0.086736 \\
\hline
\end{tabular}

TABLE 2: Water as a function of temperature (White [38]).

\begin{tabular}{lcc}
\hline$T_{1}(C)$ & $\rho_{1}\left(\mathrm{~kg} / \mathrm{m}^{3}\right)$ & $\mu_{1} \times 10^{-3} \mathrm{Ns} / \mathrm{m}^{2}$ \\
\hline 0 & 1000 & 1.788 \\
10 & 1000 & 1.307 \\
20 & 998 & 1.003 \\
30 & 996 & 0.799 \\
40 & 992 & 0.657 \\
50 & 988 & 0.548 \\
60 & 983 & 0.467 \\
70 & 978 & 0.405 \\
80 & 972 & 0.355 \\
90 & 965 & 0.316 \\
100 & 958 & 0.283 \\
\hline
\end{tabular}

where $\gamma$ is a fluid property. Hereafter, the subscript " $r$ " denotes reference value. When $T_{o} \approx T_{r}$, we obtain

$$
\mu_{1}=\frac{\mu_{o}}{1-T_{1}-T_{o} / \theta_{r}\left(T_{w}-T_{o}\right)}=\frac{\mu_{o}}{1-\widetilde{\theta}(\eta) / \theta_{r}}
$$

where $\theta_{r}=-1 / \gamma\left(T_{w}-T_{o}\right)$ is the fluid viscosity parameter. Substituting equation (19) into (10), we get

$$
\begin{aligned}
& \frac{\theta_{r}}{(\theta r-\tilde{\theta})} \widetilde{f}^{\prime \prime \prime}+\frac{\tilde{f}^{\prime \prime} \tilde{\theta}^{\prime} \theta_{r}}{\left(\theta_{r}-\tilde{\theta}\right)^{2}}+N \tilde{f}^{\prime \prime \prime}-N \lambda\left(\frac{n+1}{2}\right) \tilde{f}^{\prime \prime 2} \widetilde{f}^{\prime \prime \prime} \\
& -\frac{2 n}{n+1} \widetilde{f}^{\prime 2}+\widetilde{f} \widetilde{f}^{\prime \prime}+M\left(E-\widetilde{f}^{\prime}\right)-K_{p} \frac{\theta_{r}}{\theta_{r}-\tilde{\theta}} \widetilde{f}^{\prime}+G_{r} \widetilde{\theta}=0 .
\end{aligned}
$$

Similarly, the variable thermal conductivity is articulated by varying temperature as [21]

$$
k_{1}\left(T_{1}\right)=k_{o}(1+\varepsilon \tilde{\varepsilon}) .
$$

Introducing equation (21) into (11), one obtains

$$
\begin{aligned}
& \left(1+\frac{4}{3} \operatorname{Rd}\right)(1+\varepsilon \tilde{\theta}) \tilde{\theta}^{\prime \prime}+\varepsilon\left(\widetilde{\theta}^{\prime}\right)^{2}+\operatorname{Pr}_{o}\left(\tilde{f} \widetilde{\theta}^{\prime}+\operatorname{Nb} \widetilde{\theta}^{\prime} \widetilde{\phi}^{\prime}+\operatorname{Nt}\left(\widetilde{\theta}^{\prime}\right)^{2}\right. \\
& \left.+\operatorname{MEc}\left(\widetilde{f}^{\prime}-E\right)^{2}+\frac{2}{n+1} s \tilde{\theta}\right)=0 .
\end{aligned}
$$

The skin friction coefficient $C_{f}$ is determined in the following manner: 


$$
C_{f}=\frac{\tau_{w}}{\rho_{1} U_{w}^{2}}
$$

in which $\tau_{w}$ is the wall shear stress defined as

$$
\tau_{w}=\left(\mu_{1} \frac{\partial u_{1}}{\partial y_{1}}+\frac{1}{\beta_{1} d} \frac{\partial u_{1}}{\partial y_{1}}-\frac{1}{6 \beta_{1} d^{3}}\left(\frac{\partial u_{1}}{\partial y_{1}}\right)^{3}\right) .
$$

Using equation (24) in (23), the skin friction coefficient in dimensionless form is defined in the following section.

3.3. The Skin Friction Coefficients (Cases A and B). The skin friction coefficients for Cases A and B are written as

$$
\begin{aligned}
& \frac{C_{f} \sqrt{\mathrm{Re}_{x}}}{2}=\sqrt{\frac{n+1}{2}}\left((1+N) \tilde{f} \prime \prime(0)-\frac{n+1}{2} \frac{N \lambda}{3}(\tilde{f} \prime \prime(0))^{3}\right), \\
& \frac{C_{f} \sqrt{\mathrm{Re}_{x}}}{2}=\sqrt{\frac{n+1}{2}}\left(\left(\frac{\theta_{r}}{\theta_{r}-\theta(0)}+N\right) \tilde{f} \prime \prime(0)-\frac{n+1}{2} \frac{N \lambda}{3}(\tilde{f} \prime(0))^{3}\right) .
\end{aligned}
$$

Likewise, we define the local Nusselt and the Sherwood numbers in the following manner.

\subsection{The Local Nusselt Number}

$$
\mathrm{Nu}_{x}=-\frac{\left(x_{1}+b\right) q_{w}}{k_{o}\left(T_{w}-T_{\infty}\right)}=-\left(1+\frac{4}{3} \mathrm{Rd}\right) \sqrt{\frac{(1+n) \mathrm{Re}_{x}}{2}} \widetilde{\theta} \prime(0) .
$$

\subsection{The Local Sherwood Number}

$$
S h_{x}=-\frac{x j_{w}}{C_{w}-C_{\infty}}=-\sqrt{\frac{(1+n) R e_{x}}{2}} \widetilde{\phi}^{\prime}(0),
$$

where $\mathrm{Re}_{x}$ is the local Reynolds number.

\section{Simplified Finite Difference Method (SFDM)}

The simplified finite difference method (SFDM) has been introduced in [45]. This scheme is motivated from the work by $\mathrm{Na}$ [46]. The algorithmic steps involved in the SFDM are as follows:
(1) Reduction of higher-order ODE into a system of first- and second-order ODEs.

(2) Linearization of nonlinear ODE through the use of Taylor series.

(3) Use of finite differences to discretize the linear second-order ODE.

(4) Finally, the obtained algebraic system is solved efficiently by LU decomposition.

(5) Repeating the above procedure will produce solutions in $\tilde{\theta}$ and $\widetilde{\phi}$.

The summary of the involved steps in the SFDM is also shown in Figure 2. The results are computed for $N=1000$ grid points in the $\eta$ direction. However, the number of grid points was varied in some calculations to achieve better accuracy. The iterative procedure has been done with tolerance of machine epsilon in MATLAB. Assuming $\widetilde{f}^{\prime}=F$ in equation (15), we may write

$$
\begin{aligned}
\frac{\mathrm{d}^{2} F}{\mathrm{~d} \eta^{2}}= & \left(\frac{1}{1+N-N \lambda(n+1 / 2)(\mathrm{d} F / \mathrm{d} \eta)^{2}}\right) \\
& \cdot\left(\frac{2 n}{n+1} F^{2}-\tilde{f} \frac{\mathrm{d} F}{\mathrm{~d} \eta}-M(E-F)+K_{p} F-G_{r} \theta\right) .
\end{aligned}
$$

Then, this expression is written as

$$
\begin{aligned}
\chi\left(h, F, F^{\prime}\right)= & \left(\frac{1}{1+N-N \lambda(n+1 / 2)(\mathrm{d} F / \mathrm{d} \eta)^{2}}\right) \\
& \cdot\left(\frac{2 n}{n+1} F^{2}-\widetilde{f} \frac{\mathrm{d} F}{\mathrm{~d} \eta}-M(E-F)+K_{p} F-G_{r} \theta\right),
\end{aligned}
$$

and we replace $\mathrm{d} F / \mathrm{d} \eta$ by forward difference approximation:

$$
\begin{aligned}
\chi\left(h, F, F^{\prime}\right)= & \left(\frac{1}{1+N-N \lambda(n+1 / 2)\left(F_{j+1}-F_{j} / h\right)^{2}}\right) \\
& \cdot\left(\left(\frac{2 n}{n+1} F_{j}^{2}-\tilde{f}_{j}\left(\frac{F_{j+1}-F_{i}}{h}\right)-M\left(E-F_{j}\right)+K_{p} F_{j}\right)-\mathrm{Gr} \theta\right) .
\end{aligned}
$$

The coefficients of second-order ODE are 


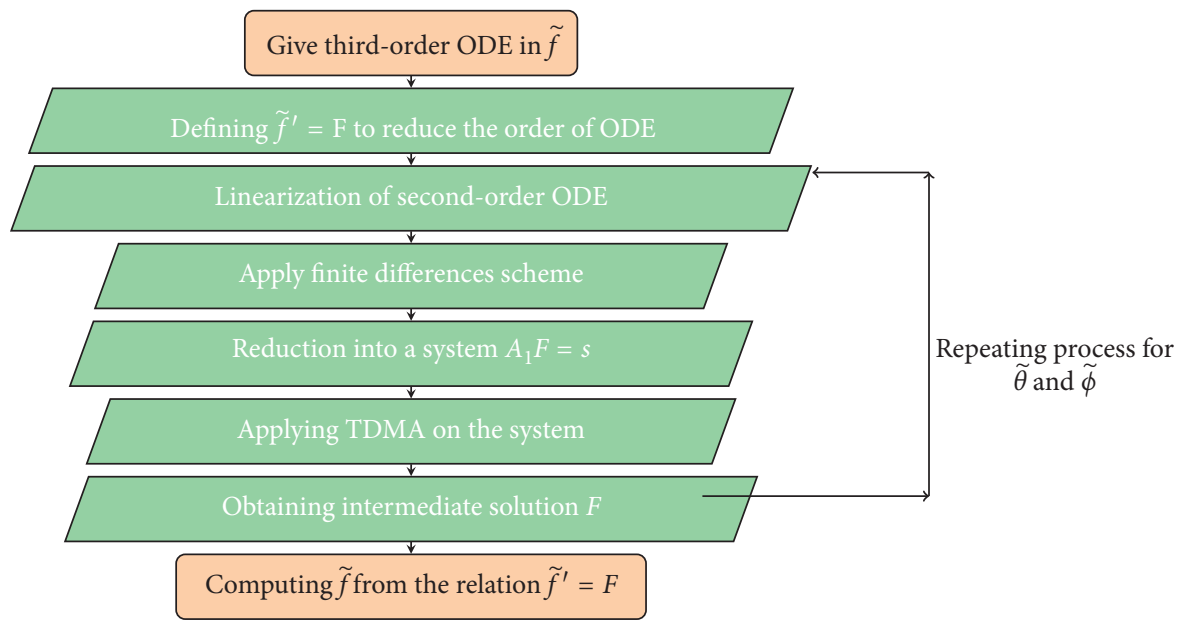

FIGURE 2: Flow diagram of SFDM [45].

$$
\begin{aligned}
& Q_{n}=-\frac{\partial \chi}{\partial F^{\prime}}=\left(\frac{1}{\left(1+N-N \lambda(n+1 / 2)(\mathrm{d} F / \mathrm{d} \eta)^{2}\right)^{2}}\right)\left[(\lambda N(n+1))\left(\frac{-2 n}{n+1} F^{2}+\tilde{f} \frac{\mathrm{d} F}{\mathrm{~d} \eta}+M(E-F)-K_{p} F+\mathrm{Gr} \theta\right)\right. \\
&\left.-\tilde{f}\left(1+N-N \lambda\left(\frac{n+1}{2}\right)\left(\frac{\mathrm{d} F}{\mathrm{~d} \eta}\right)^{2}\right)\right]=\left(\frac{1}{\left(1+N-N \lambda(n+1 / 2)\left(F_{j+1}-F_{j} / h\right)^{2}\right)^{2}}\right) \\
& \cdot\left[\lambda N(n+1)\left(\frac{-2 n}{n+1} F_{j}^{2}+\tilde{f}_{j} \frac{F_{j+1}-F_{j}}{h}+M(E-F)-K_{p} F_{j}+G r \theta\right)-f_{j}\left(1+N-N \lambda\left(\frac{n+1}{2}\right)\left(\frac{F_{j+1}-F_{j}}{h}\right)\right),\right. \\
& R_{n}=-\frac{\partial \chi}{\partial F}=-\left(\frac{1}{1+N-N \lambda(n+1 / 2)(\mathrm{d} F / \mathrm{d} \eta)^{2}}\right)\left(\frac{4 n}{n+1} F+M+\mathrm{Kp}\right), \\
&=\left(\frac{1}{1+N-N \lambda(n+1 / 2)\left(F_{j+1}-F_{i} / h\right)^{2}}\right)\left(\frac{4 n}{n+1} F_{j}+M+\mathrm{Kp}\right), \\
& S_{n}=\chi\left(h, F, F^{\prime}\right)+R_{n} F_{j}+Q_{n} \frac{F_{j+1}-F_{j}}{h} .
\end{aligned}
$$

After manipulating equations (28)-(33), the linear algebraic system in $F$ is written as [45]

$$
X_{j} F_{j-1}+Y_{j} F_{j}+Z_{j} F_{j+1}=W_{j}, \quad j=1,2,3, \ldots, N,
$$

where the coefficients are defined by

$$
\begin{aligned}
X_{j} & =2-h Q_{n}, \\
Y_{j} & =2 h^{2} R_{n}-4, \\
Z_{j} & =2+h Q_{n}, \\
W_{j} & =2 h^{2} S_{n} .
\end{aligned}
$$


In matrix-vector format, it is

$$
A_{1} F=S \text {, }
$$

where

$$
A_{1}=\left[\begin{array}{ccccccc}
Y_{1} & Z_{1} & & & & \\
X_{2} & Y_{2} & Z_{2} & & & & \\
& & \cdots & & & \\
& & & X_{N-2} & Y_{N-2} & Z_{N-2} \\
& & & & X_{N-1} & Y_{N-1}
\end{array}\right]
$$

is a tridiagonal matrix. The column vectors $F$ and $s$ are

$$
\begin{gathered}
F=\left[\begin{array}{c}
F_{1} \\
F_{2} \\
\cdot \\
\cdot \\
F_{N-1}
\end{array}\right], \\
s=\left[\begin{array}{c}
s_{1} \\
s_{2} \\
\cdot \\
\cdot \\
s_{N-1}
\end{array}\right] .
\end{gathered}
$$

4.1. Thomas Algorithm. The Thomas algorithm [47] is implemented in MATLAB to compute the solution $F$. LU factorization is chosen for the matrix factorization of $A_{1}$, i.e.,

$$
A_{1}=L_{1} U_{1} \text {, }
$$

where

$$
\begin{aligned}
L_{1} & =\left[\begin{array}{llllll}
\Lambda_{1} & & & & & \\
X_{2} & \Lambda_{2} & & & & \\
& & \ldots & & & \\
& & & X_{N-2} & \Lambda_{N-2} & \\
& & & & X_{N-1} & \Lambda_{N-1}
\end{array}\right], \\
U_{1} & =\left[\begin{array}{cccccc}
1 & \zeta_{1} & & & & \\
& 1 & \zeta_{2} & & & \\
& & \ldots & & & \\
& & & 1 & \zeta_{N-2} \\
& & & & 1
\end{array}\right] .
\end{aligned}
$$

It is clear that $L_{1}$ and $U_{1}$ are the lower and unit upper triangular matrices, respectively. Variables $\left(\Lambda_{j}, \zeta_{j}\right), \quad j=1,2, \ldots, N-1$, are related by

$$
\begin{aligned}
& \Lambda_{1}=-1-\frac{\lambda}{h}, \\
& \zeta_{1}=\frac{\lambda}{\Lambda_{1} h}, \\
& \Lambda_{j}=Y_{j}-X_{j} \zeta_{j-1}, \quad j=2,3, \ldots, N-1,
\end{aligned}
$$

$$
\Lambda_{j} \zeta_{j}=z_{j}, \quad j=2,3, \ldots, N-2
$$

After defining these relations, equation (36) becomes

$$
\begin{array}{r}
L_{1} U_{1} F=S, \\
U_{1} F=z, \\
L_{1} z=S .
\end{array}
$$

We have

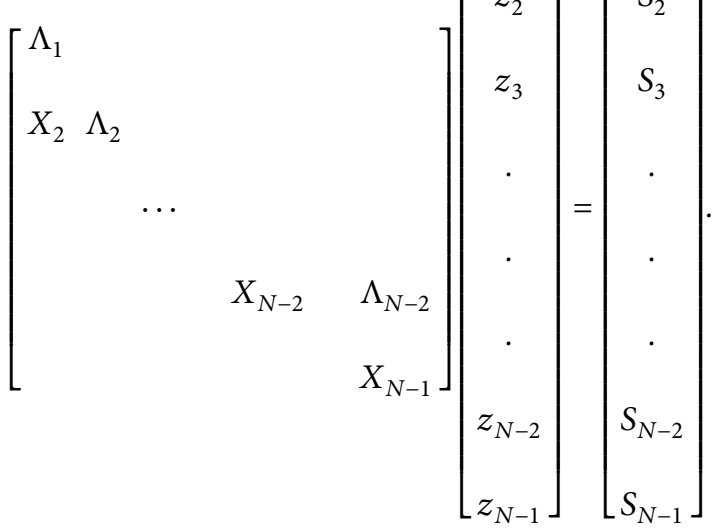


TABLE 3: Numerical values of $-C_{f} \operatorname{Re}_{x}^{1 / 2}$ for several values of involved parameters $n, \lambda, M, N$, and $\alpha$ when $\mathrm{Nb}=s=0.1, \operatorname{Pr}_{o}=1, \mathrm{Le}=1$, and $\mathrm{Nt}=\mathrm{Ec}=\mathrm{Rd}=0.2$.

\begin{tabular}{|c|c|c|c|c|c|c|c|c|c|c|}
\hline$M$ & $\mathrm{~N}$ & $\lambda$ & $\alpha$ & $N$ & $\mathrm{Gr}$ & $E$ & $K_{p}$ & $\theta_{r}$ & $\begin{array}{c}\text { Case A } \\
-C_{f} \mathrm{Re}_{x}^{1 / 2}\end{array}$ & $\begin{array}{c}\text { Case B } \\
-C_{f} \mathrm{Re}_{x}^{1 / 2}\end{array}$ \\
\hline 0.0 & 0.5 & 0.2 & 0.3 & 0.5 & 0 & 0 & 0.2 & -10 & 2.1927 & 2.1231 \\
\hline 0.4 & & & & & & & & & 2.5751 & 2.4938 \\
\hline 0.6 & & & & & & & & & 2.7458 & 2.6588 \\
\hline \multirow[t]{3}{*}{0.3} & 0.4 & 0.2 & 0.3 & 0.5 & 0 & 0 & 0.2 & -10 & 2.3729 & 2.2989 \\
\hline & 0.6 & & & & & & & & 2.5925 & 2.5097 \\
\hline & 0.8 & & & & & & & & 2.7939 & 2.7033 \\
\hline \multirow[t]{3}{*}{0.3} & 0.5 & 0.0 & 0.3 & 0.5 & 0 & 0 & 0.2 & -10 & 2.4957 & 2.4183 \\
\hline & & 0.3 & & & & & & & 2.4798 & 2.4008 \\
\hline & & 0.6 & & & & & & & 2.4630 & 2.3821 \\
\hline \multirow[t]{3}{*}{0.3} & 0.5 & 0.2 & 0.0 & 0.5 & 0 & 0 & 0.2 & -10 & 2.3954 & 2.3154 \\
\hline & & & 0.2 & & & & & & 2.4550 & 2.3760 \\
\hline & & & 0.4 & & & & & & 2.5158 & 2.4379 \\
\hline \multirow[t]{3}{*}{0.3} & 0.5 & 0.2 & 0.3 & 0.0 & 0 & 0 & 0.2 & -10 & 2.0547 & 1.9586 \\
\hline & & & & 0.3 & & & & & 2.3219 & 2.2378 \\
\hline & & & & 0.5 & & & & & 2.4852 & 2.4068 \\
\hline \multirow[t]{3}{*}{0.3} & 0.5 & 0.2 & 0.3 & 0.5 & 0.2 & 0 & 0.2 & -10 & 2.2394 & 2.1527 \\
\hline & & & & & 0.4 & & & & 2.0119 & 1.9198 \\
\hline & & & & & 0.6 & & & & 1.7955 & 1.6989 \\
\hline \multirow[t]{3}{*}{0.3} & 0.5 & 0.2 & 0.3 & 0.5 & 0 & 0.2 & 0.2 & -10 & 2.3220 & 2.2484 \\
\hline & & & & & & 0.4 & & & 2.1687 & 2.0984 \\
\hline & & & & & & 0.7 & & & 1.9511 & 1.8844 \\
\hline \multirow[t]{3}{*}{0.3} & 0.5 & 0.2 & 0.3 & 0.5 & 0 & 0 & 0.5 & -10 & 2.7458 & 2.6403 \\
\hline & & & & & & & 1 & & 3.1300 & 2.9840 \\
\hline & & & & & & & 1.5 & & 3.4692 & 3.2834 \\
\hline \multirow[t]{3}{*}{0.3} & 0.5 & 0.2 & 0.3 & 0.5 & 0 & 0 & 0.2 & -5 & & 2.3380 \\
\hline & & & & & & & & -3 & & 2.2586 \\
\hline & & & & & & & & -1 & & 1.9935 \\
\hline
\end{tabular}

TABle 4: Numerical values of $-\mathrm{Nu}_{x} \mathrm{Re}_{x}^{-1 / 2}$ for several values of involved parameters $n, M, \alpha, \mathrm{Nb}, \mathrm{Nt}, \mathrm{Pr}$, and Le when $\mathrm{Gr}=0$, $\mathrm{Kp}=\mathrm{Ec}=\lambda=0.2, N=0.5$, and $\theta_{r}=-10$.

\begin{tabular}{|c|c|c|c|c|c|c|c|c|c|c|c|c|}
\hline$M$ & $n$ & $\operatorname{Pr}_{o}$ & Le & $N_{t}$ & $N_{b}$ & $\alpha$ & $\mathrm{Rd}$ & $E$ & $s$ & $\epsilon$ & $\begin{array}{c}\text { Case A } \\
-\mathrm{Nu}_{x} \mathrm{Re}_{x}^{-1 / 2}\end{array}$ & $\begin{array}{c}\text { Case B } \\
-\mathrm{Nu}_{x} \mathrm{Re}_{x}^{-1 / 2}\end{array}$ \\
\hline 0.0 & 0.5 & 1 & 1 & 0.2 & 0.1 & 0.3 & 0.2 & 0 & 0.1 & 0.2 & 0.4801 & 0.4028 \\
\hline 0.3 & & & & & & & & & & & 0.4158 & 0.3430 \\
\hline 0.7 & & & & & & & & & & & 0.3365 & 0.2673 \\
\hline \multirow[t]{3}{*}{0.3} & 0.2 & 1 & 1 & 0.2 & 0.1 & 0.3 & 0.2 & 0 & 0.1 & 0.2 & 0.4055 & 0.3326 \\
\hline & 0.5 & & & & & & & & & & 0.4157 & 0.3430 \\
\hline & 1 & & & & & & & & & & 0.4427 & 0.3685 \\
\hline \multirow[t]{3}{*}{0.3} & 0.5 & 0.7 & 1 & 0.2 & 0.1 & 0.3 & 0.2 & 0 & 0.1 & 0.2 & 0.2742 & 0.2094 \\
\hline & & 1 & & & & & & & & & 0.4157 & 0.3430 \\
\hline & & 1.3 & & & & & & & & & 0.5268 & 0.4427 \\
\hline \multirow[t]{3}{*}{0.3} & 0.5 & 1 & 0.7 & & 0.1 & 0.3 & 0.2 & 0 & 0.1 & 0.2 & 0.4187 & 0.3453 \\
\hline & & & 1.0 & & & & & & & & 0.4157 & 0.3430 \\
\hline & & & 1.3 & & & & & & & & 0.4136 & 0.3413 \\
\hline \multirow[t]{3}{*}{0.3} & 0.5 & 1 & 1.0 & 0 & 0.1 & 0.3 & 0.2 & 0 & 0.1 & 0.2 & 0.4362 & 0.3610 \\
\hline & & & & 0.2 & & & & & & & 0.4157 & 0.3430 \\
\hline & & & & 0.4 & & & & & & & 0.3952 & 0.3250 \\
\hline \multirow[t]{3}{*}{0.3} & 0.5 & 1 & & 0.2 & 0.2 & 0.3 & 0.2 & 0 & 0.1 & 0.2 & 0.4157 & 0.3430 \\
\hline & & & & & 0.5 & & & & & & 0.4157 & 0.3430 \\
\hline & & & & & 0.7 & & & & & & 0.4157 & 0.3430 \\
\hline \multirow[t]{3}{*}{0.3} & 0.5 & 1 & 1.0 & 0.2 & 0.1 & 0.0 & 0.2 & 0 & 0.1 & 0.2 & 0.3469 & 0.2827 \\
\hline & & & & & & 0.4 & & & & & 0.4385 & 0.3627 \\
\hline & & & & & & 0.8 & & & & & 0.5301 & 0.4413 \\
\hline \multirow[t]{3}{*}{0.3} & 0.5 & 1 & 1.0 & 0.2 & 0.2 & 0.3 & 0.0 & 0 & 0.1 & 0.2 & 0.4092 & 0.3433 \\
\hline & & & & & & & 0.5 & & & & 0.4142 & 0.3379 \\
\hline & & & & & & & 0.8 & & & & 0.3890 & 0.2994 \\
\hline
\end{tabular}


TABle 4: Continued.

\begin{tabular}{|c|c|c|c|c|c|c|c|c|c|c|c|c|}
\hline$M$ & $n$ & $\operatorname{Pr}_{o}$ & Le & $N_{t}$ & $N_{b}$ & $\alpha$ & $\mathrm{Rd}$ & E & $s$ & $\epsilon$ & $\begin{array}{c}\text { Case A } \\
-\mathrm{Nu}_{x} \mathrm{Re}_{x}^{-1 / 2}\end{array}$ & $\begin{array}{c}\text { Case B } \\
-\mathrm{Nu}_{x} \mathrm{Re}_{x}^{-1 / 2}\end{array}$ \\
\hline \multirow[t]{3}{*}{0.3} & 0.5 & 1 & 1.0 & 0.2 & 0.1 & 0.0 & 0.2 & 0.2 & 0.1 & 0.2 & 0.4930 & 0.4121 \\
\hline & & & & & & 0.4 & & 0.4 & & & 0.4789 & 0.3942 \\
\hline & & & & & & 0.8 & & 0.7 & & & 0.4285 & 0.3420 \\
\hline \multirow[t]{3}{*}{0.3} & 0.5 & 1 & 1.0 & 0.2 & & 0.3 & 0.2 & 0 & 0 & 0.2 & 0.5699 & 0.4892 \\
\hline & & & & & & & & & 0.1 & & 0.4157 & 0.3430 \\
\hline & & & & & & & & & 0.15 & & 0.2940 & 0.2127 \\
\hline \multirow[t]{3}{*}{0.3} & 0.5 & 1 & 1.0 & 0.2 & 0.1 & 0.3 & 0.2 & 0 & 0.1 & 0 & & 0.4069 \\
\hline & & & & & & & & 0.4 & & 0.5 & & 0.2751 \\
\hline & & & & & & & & 0.8 & & 1 & & 0.2003 \\
\hline
\end{tabular}

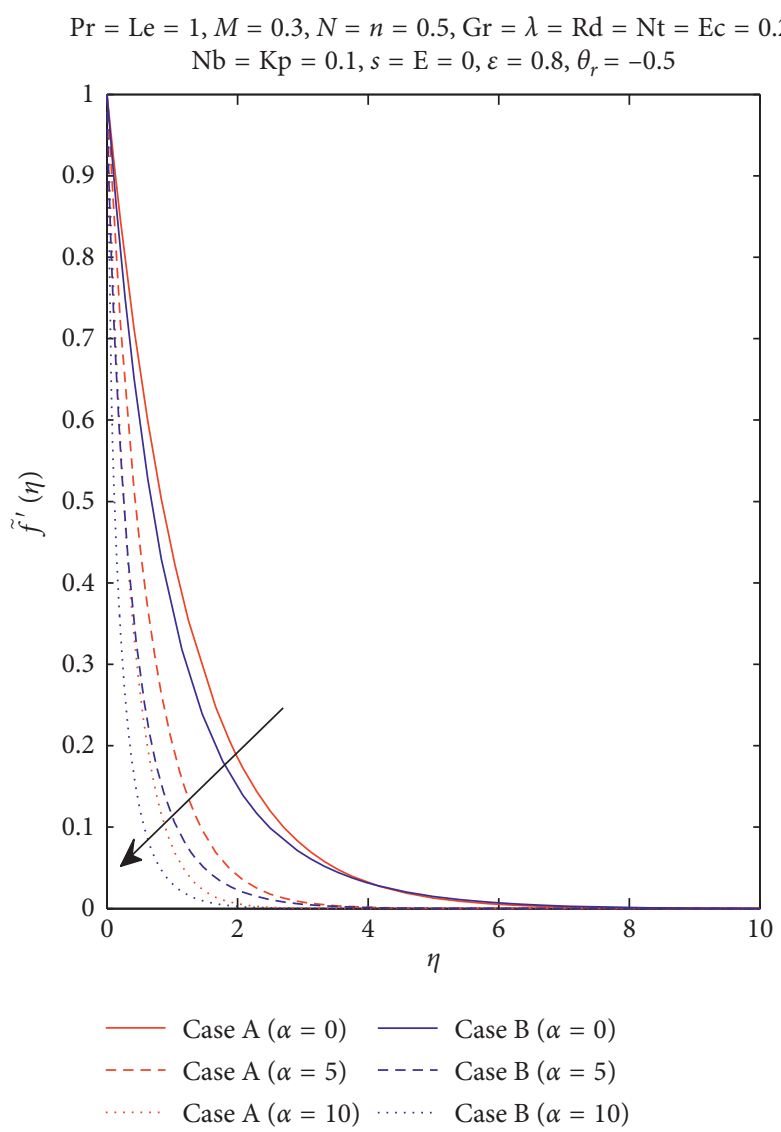

(a)

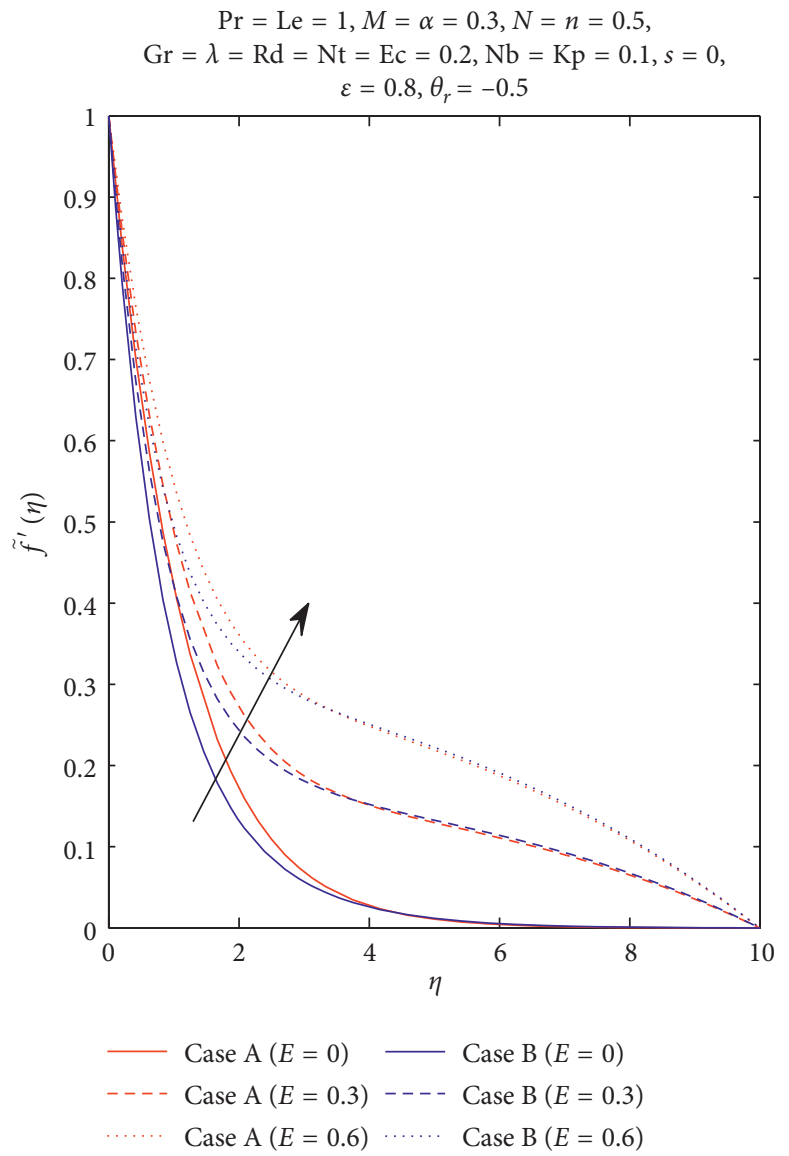

(b)

Figure 3: Continued. 


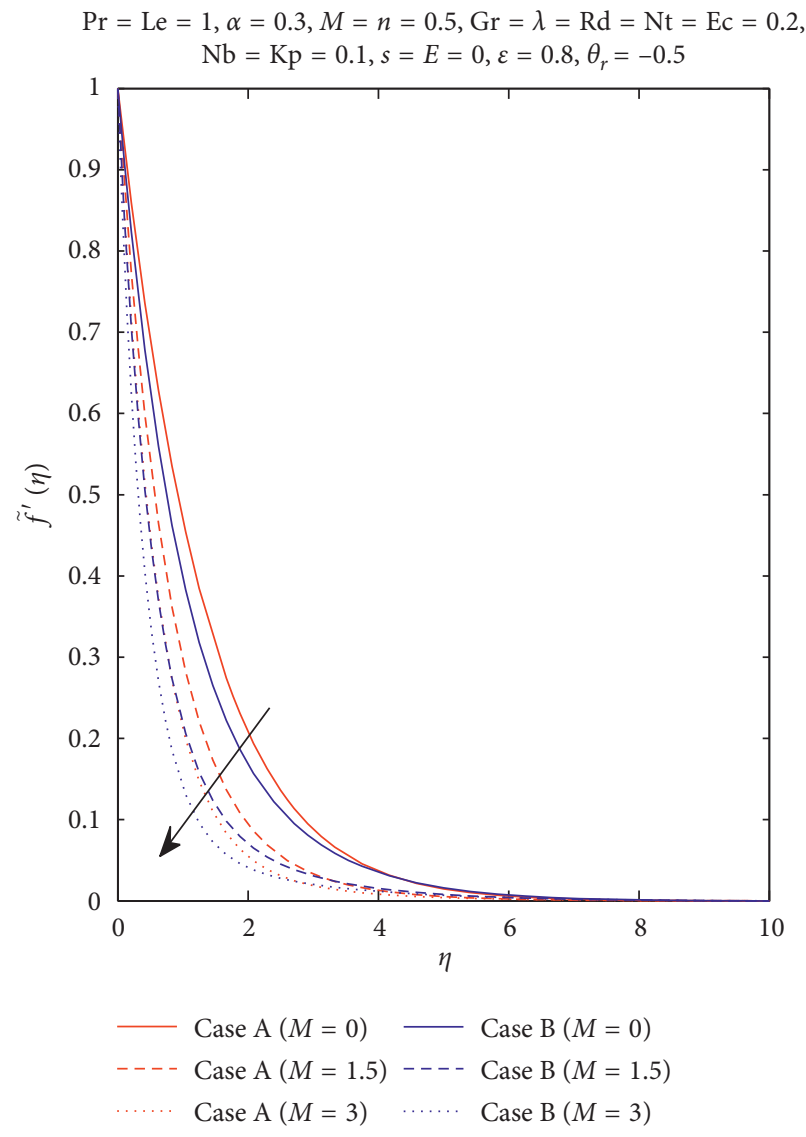

(c)

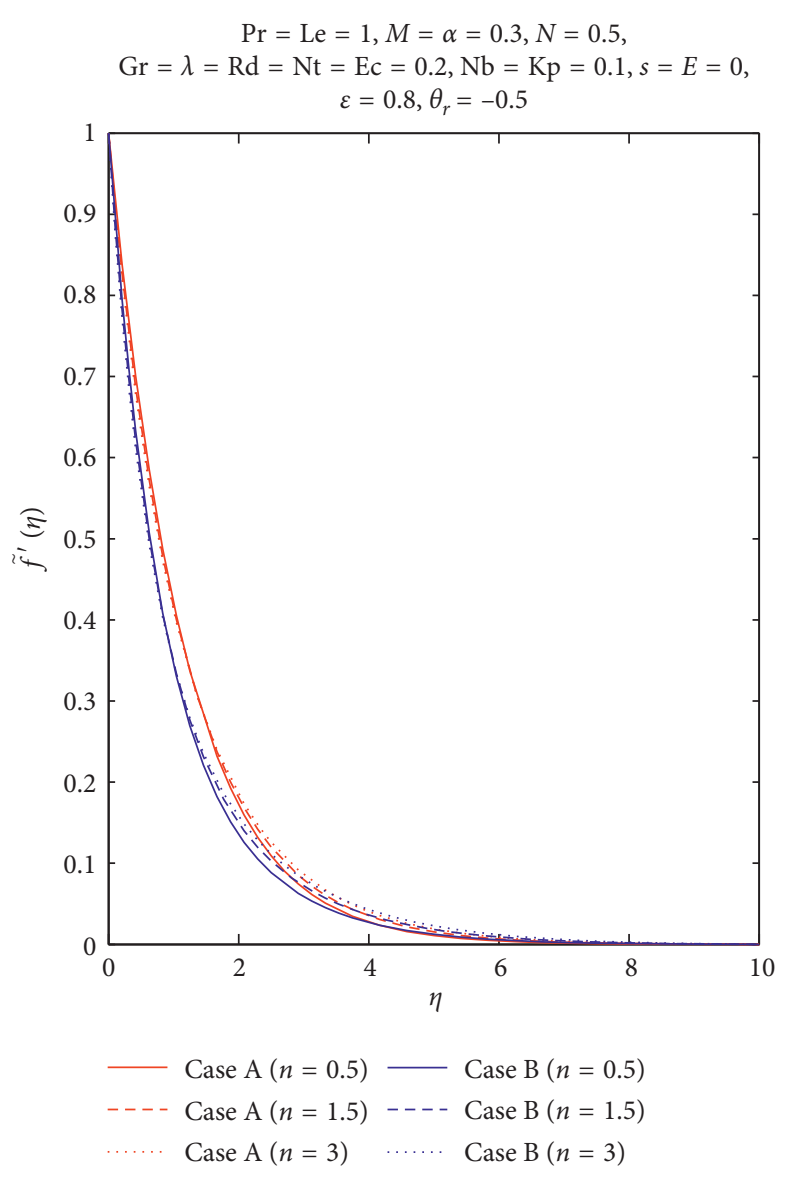

(d)

FIGURE 3: Velocity consideration supplied against (a) $\alpha$, (b) $E$, (c) $M$, and (d) $n$.

$$
\begin{aligned}
& z_{1}=\frac{S_{1}}{\Lambda_{1}} \\
& z_{j}=\frac{S_{j}-X_{j} z_{j-1}}{\Lambda_{j}}, \quad j=2,3, \ldots, N-1,
\end{aligned}
$$

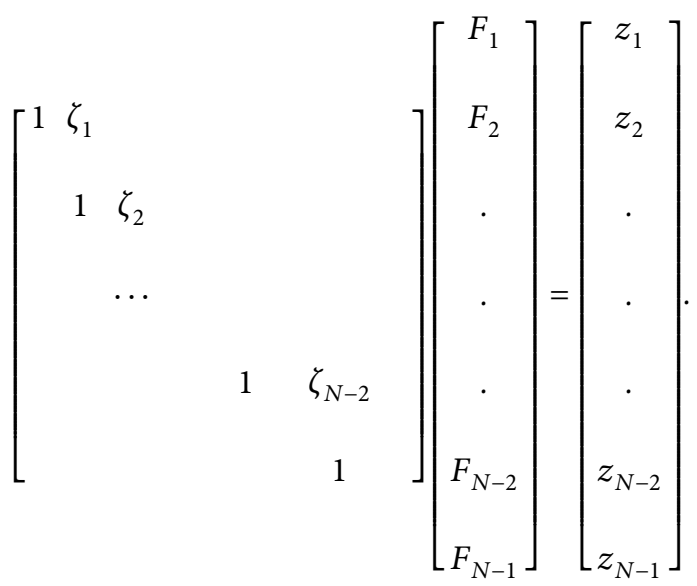




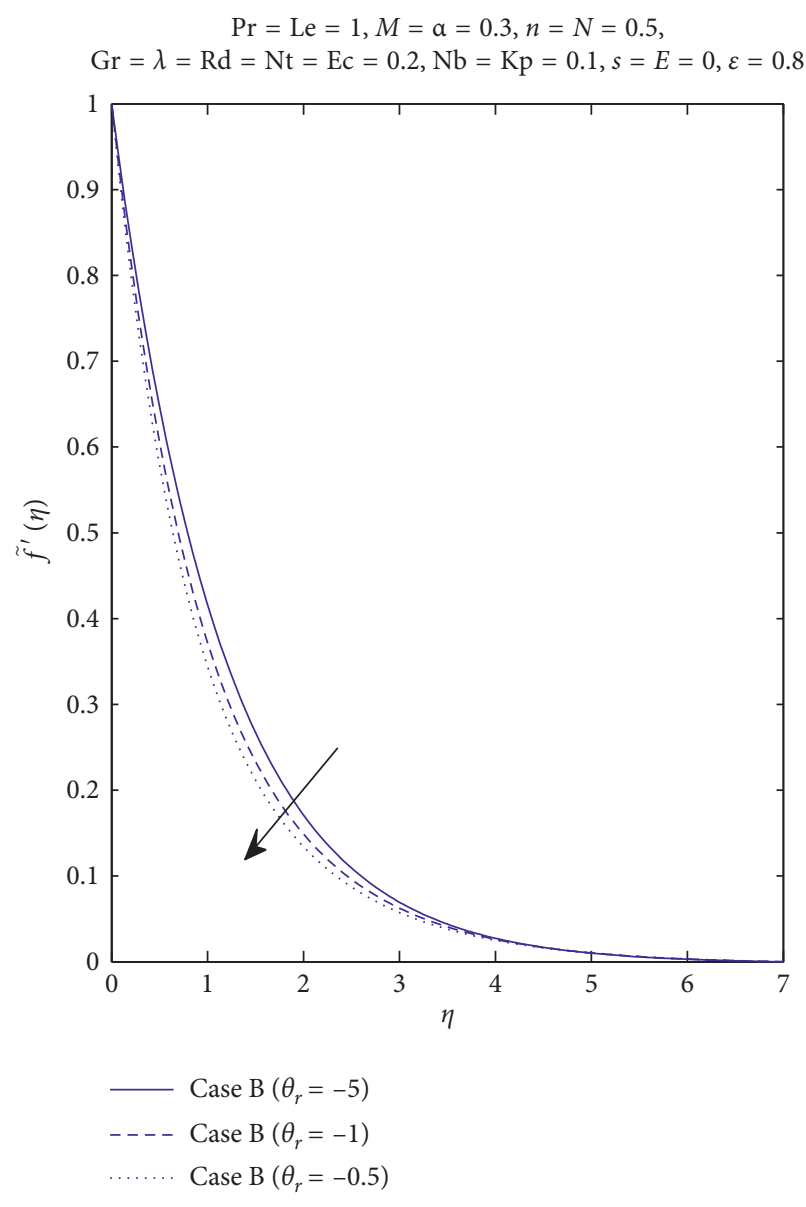

(a)

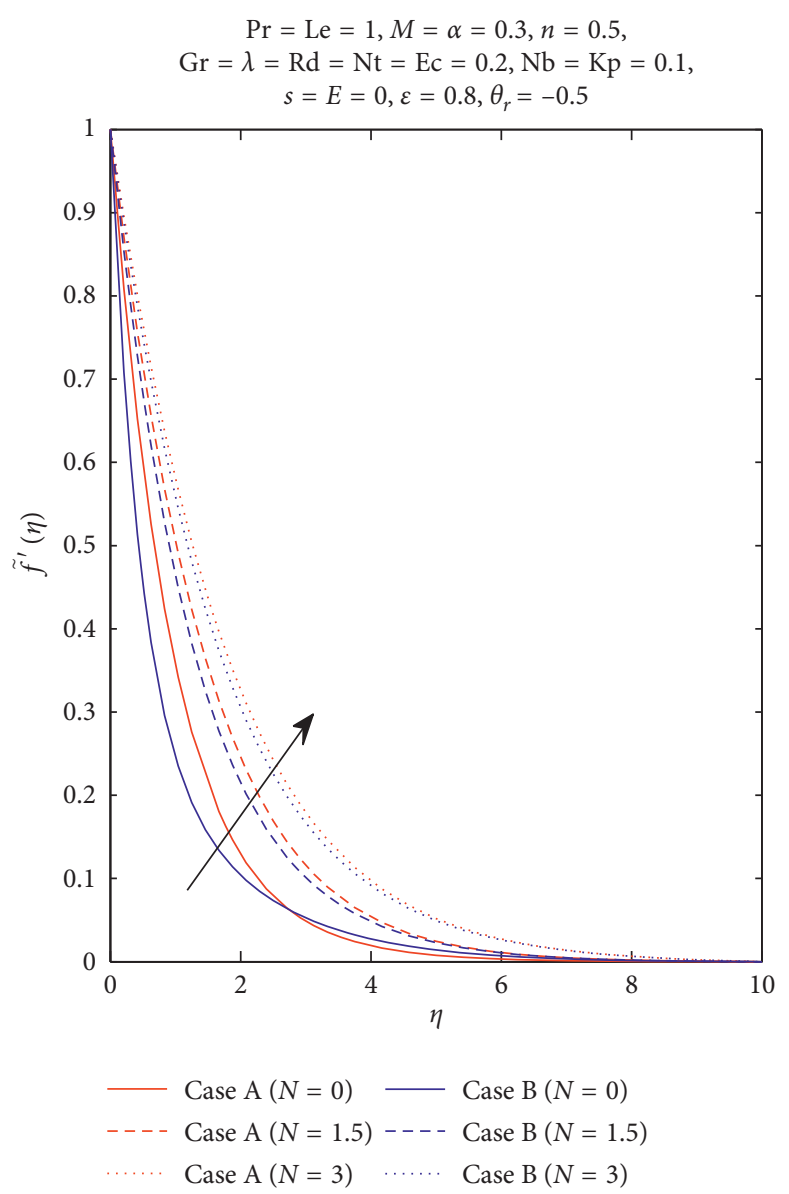

(b)

FIgURE 4: Continued. 


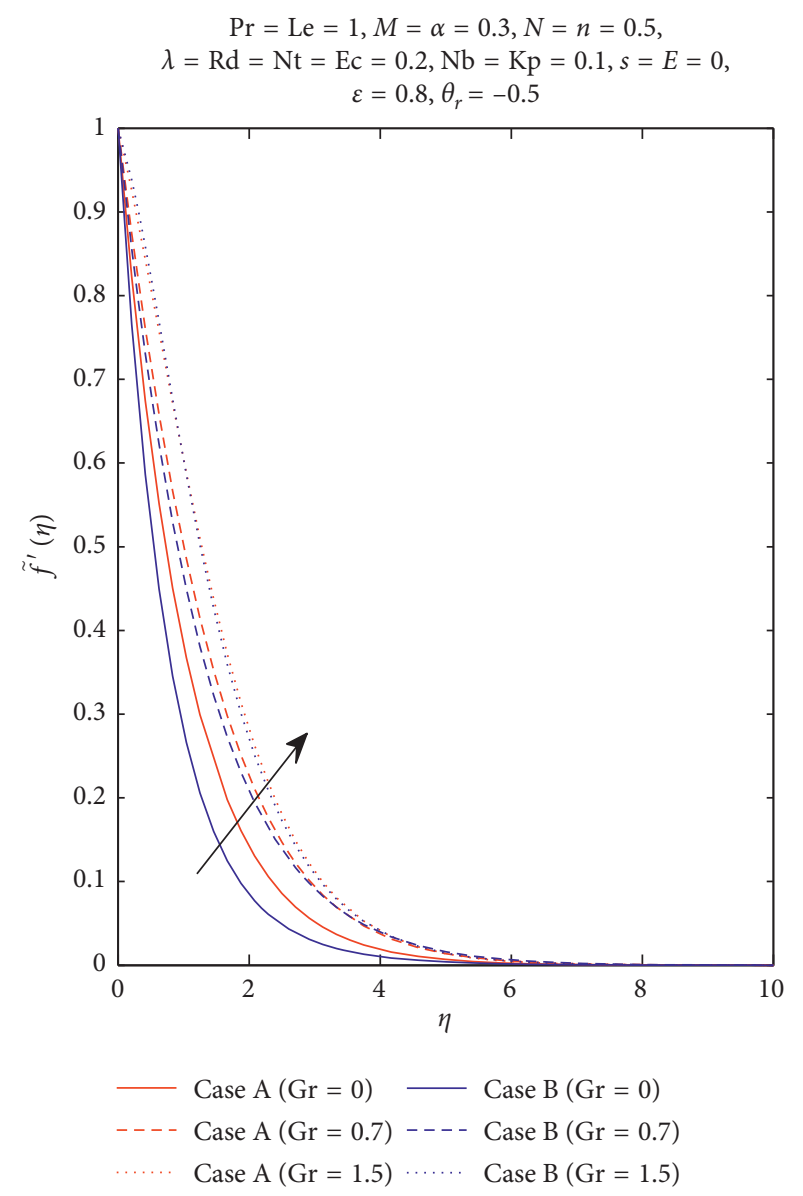

(c)

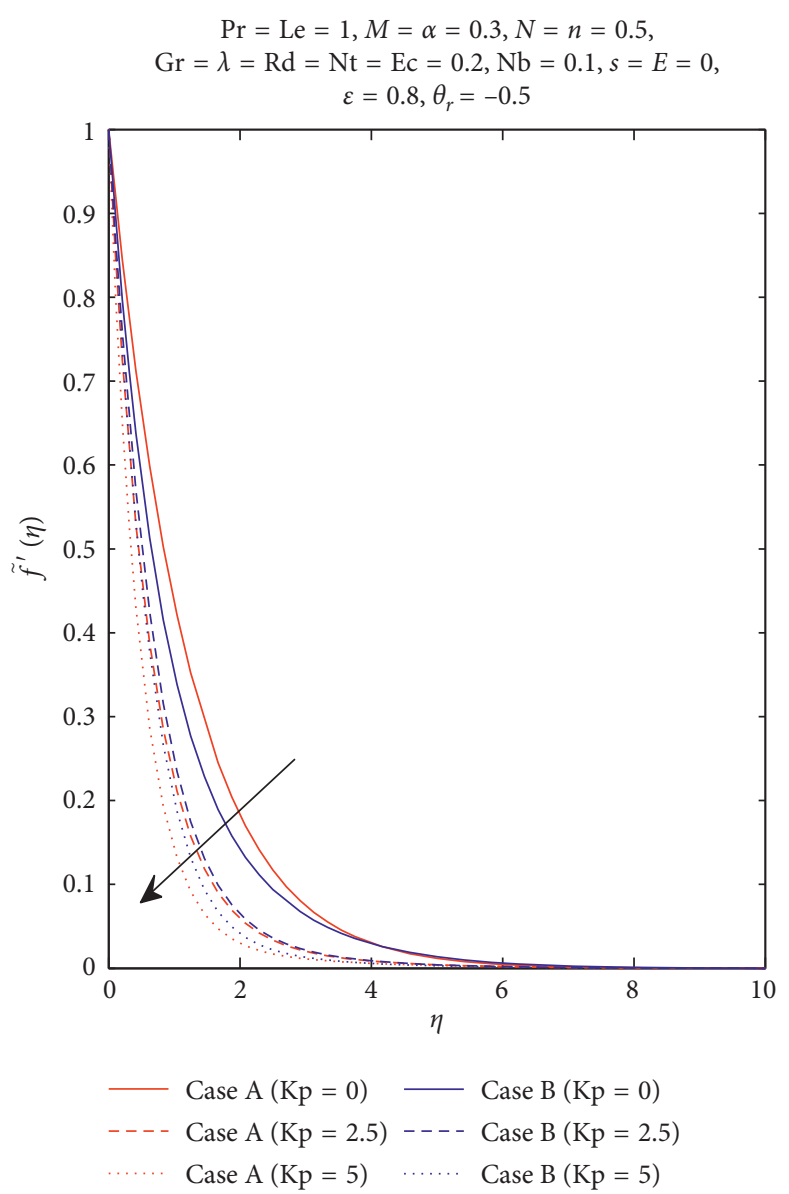

(d)

FIGURE 4: Velocity consideration supplied against (a) $\theta_{r}$, (b) $N$, (c) Gr, and (d) Kp.

We get

$$
\begin{aligned}
F_{j-1} & =z_{j-1}, \\
F_{j} & =z_{j}-\zeta_{j} F_{j+1}, \quad j=N-2, N-3, \ldots, 3,2,1 .
\end{aligned}
$$

The above equation (45) is a solution of equation (28). We discretize the relation $f^{\prime}=F$ as follows:

$$
\frac{\tilde{f}_{j+1}-\tilde{f}_{j}}{h}=F_{j},
$$

which gives a required solution of equation (15). Repeating the above process, one can easily get $\widetilde{\theta}$ and $\widetilde{\phi}$ appearing in equations (16) and (17). However, all the details for these variables have been omitted for brevity. The reader is referred to [45] for further details on the SFDM. Since the SFDM results for both constant and variable fluid properties have been discussed in detail in [45], in the current work, only Table 2 is produced from the SFDM to compare accuracy with the literature. However, we obtain the skin friction coefficient and the local Nusselt numbers for Case A and Case B
(Tables 3 and 4 ) by using MATLAB built-in solver $b v p 4 c$ cf. [48].

\section{Results and Discussion}

The comparison of skin friction coefficient for Case A and Case B is given in Table 3 . The skin friction coefficient increases for higher values of magnetic parameter $M$, power index parameter $n$, variable thickness parameter $\alpha$, power index parameter $n$, and porosity parameter $\mathrm{Kp}$ while it decreases with the increase of parameter $\lambda$, Grashof number Gr, and electric field parameter $E$. The same phenomenon is observed for the skin friction coefficient in both Cases A and B. The skin friction coefficient decreases for larger values of viscosity parameter $\theta_{r}$ in Case B. Table 4 shows the variation of local Nusselt number for Cases A and B in relation to different pertinent parameters. Note that local Nusselt number went up for larger values of power index parameter $n$ and variable thickness parameter $\alpha$ while it went down for escalating values of magnetic parameter $M$, Prandtl number $\operatorname{Pr}_{o}$, 


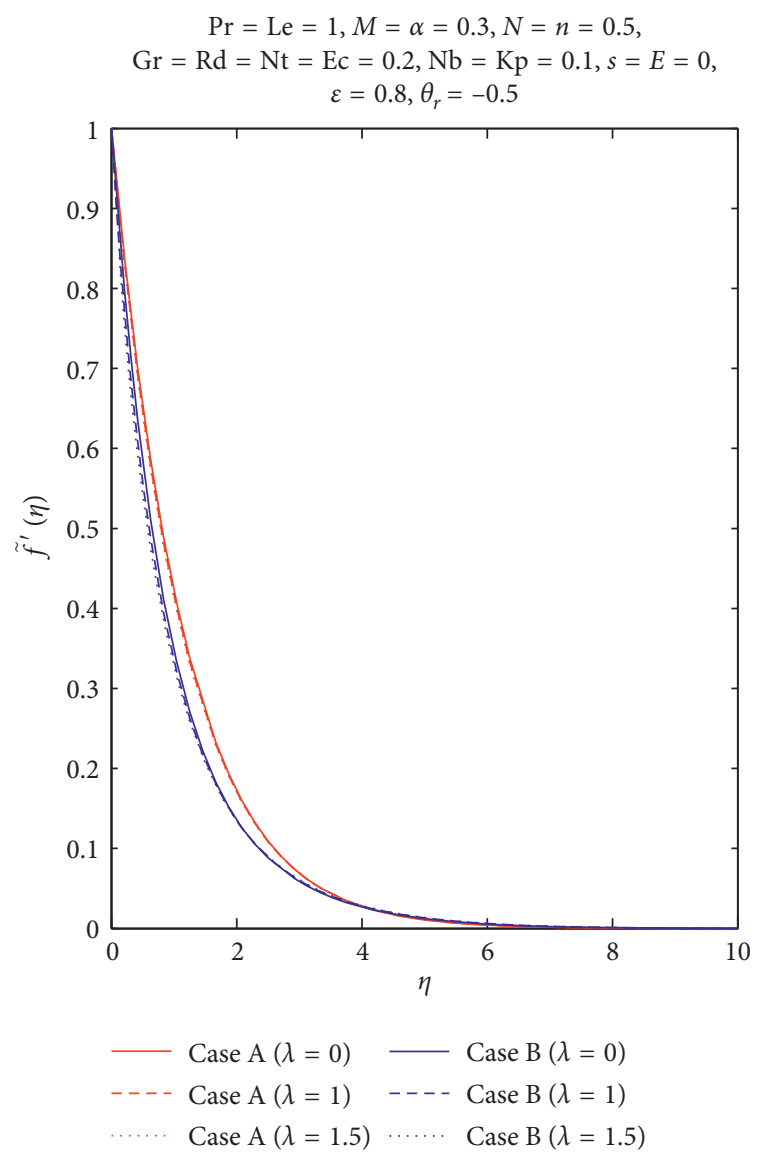

(a)

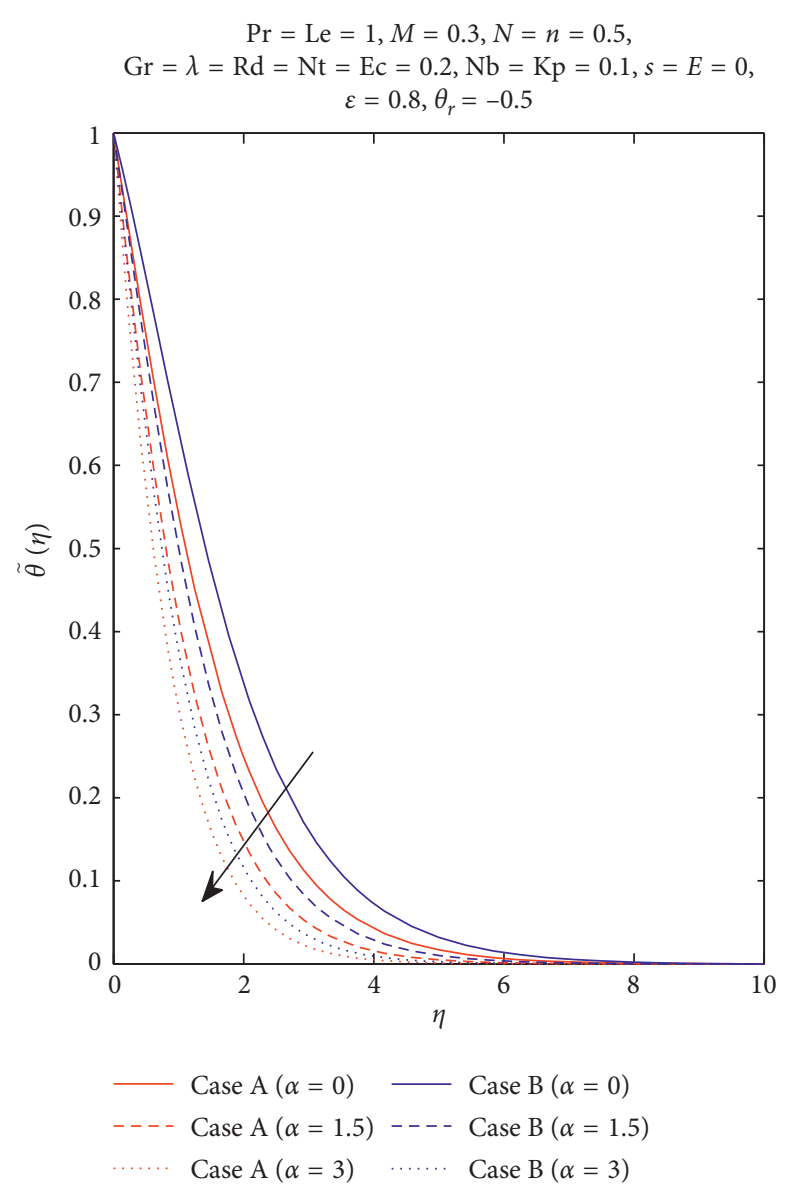

(b)

FIgURE 5: Continued. 


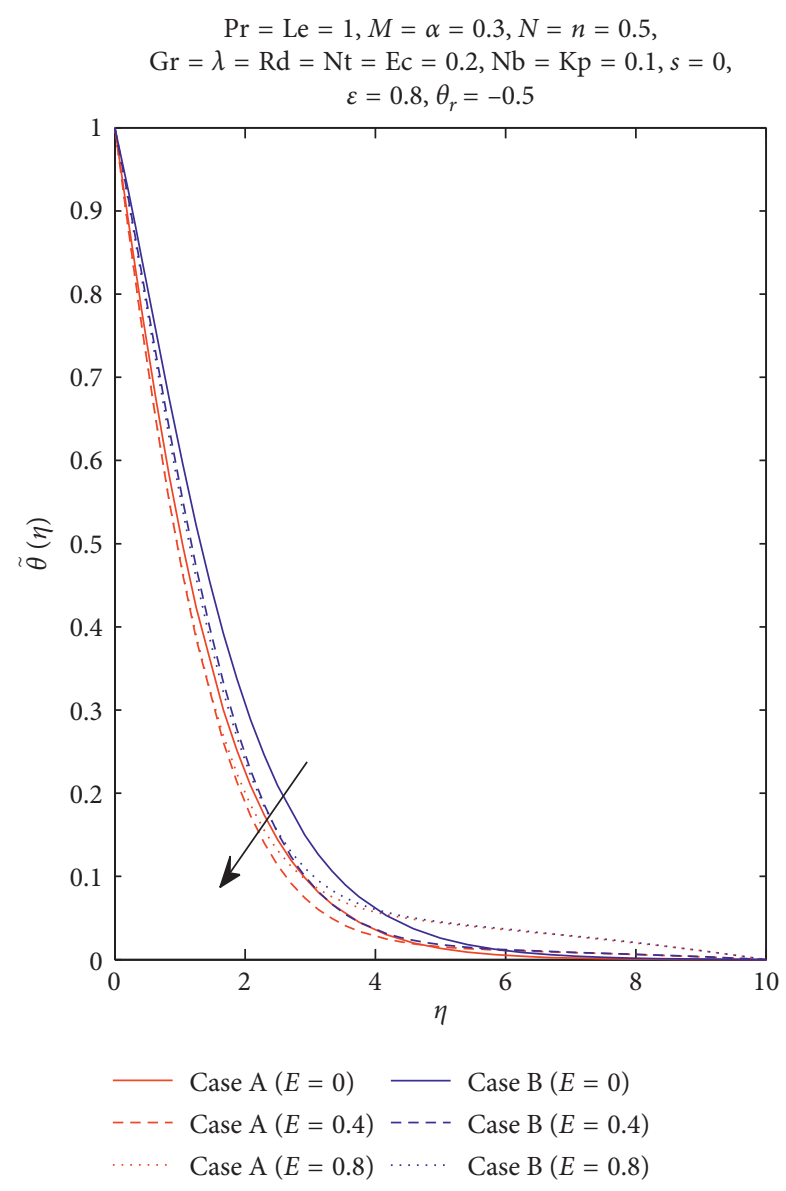

(c)

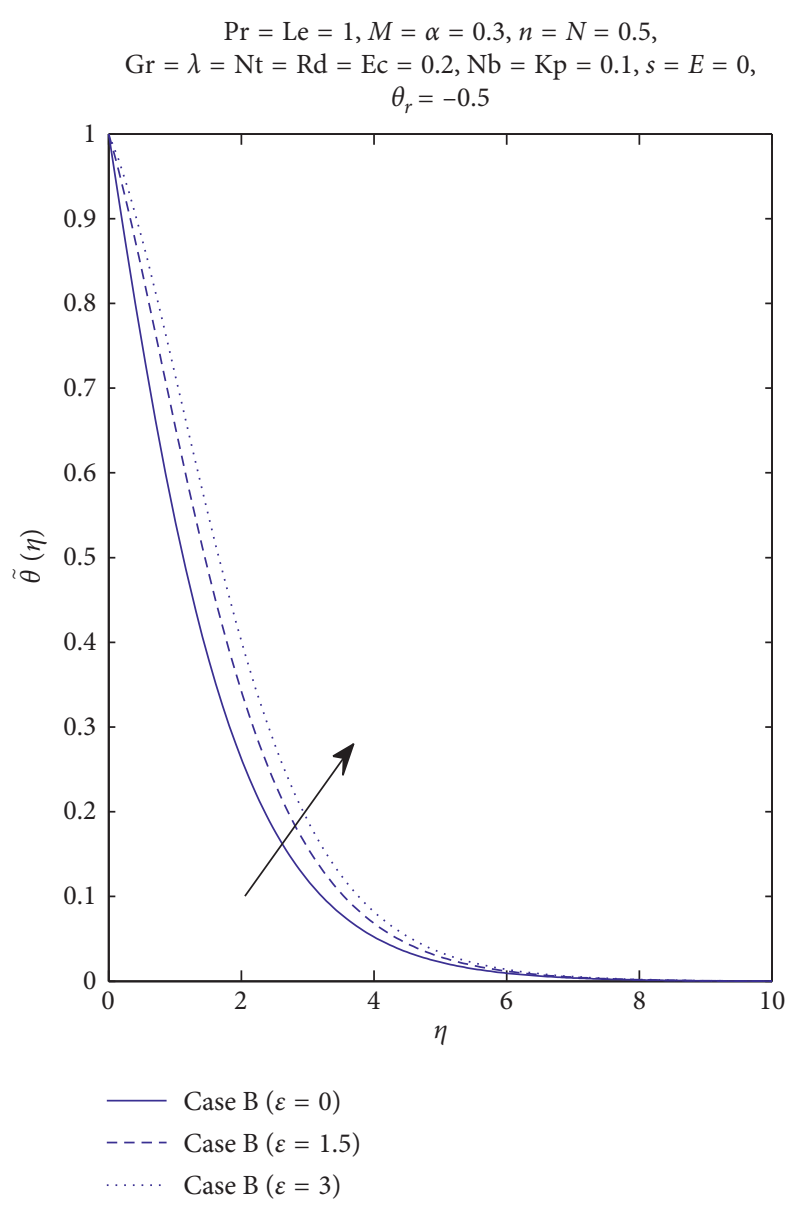

(d)

Figure 5: Velocity and temperature considerations supplied against (a) $\lambda$, (b) $\alpha$, (c) $E$, and (d) $\epsilon$.

thermophoretic parameter $\mathrm{Nt}$, radiation parameter $\mathrm{Rd}$, electric field parameter $E$, and heat source parameter $s$. The local Nusselt number for Case B decreases for larger values of small thermal conductivity parameter $\epsilon$. In these tables, we can see the difference in values for the skin friction coefficient and the local Nusselt numbers. The skin friction coefficient and the local Nusselt numbers for the results of constant fluid properties have higher values when compared with the results obtained for variable fluid properties.

Figure 3(a) describes the role of wall thickness parameter $\alpha$ in the fluid's velocity. Note that the velocity profile diminishes significantly for both Cases A and B with an increase in the values of wall thickness parameter $\alpha$. It is observed that higher values of variable thickness parameter $\alpha$ correspond to deformation due to stretching wall, and hence the velocity profile decreases. Figure $3(\mathrm{~b})$ is plotted to perceive the effect of electric field parameter $E$ on velocity distribution. It is shown that the increase in electric field parameter $E$ enhances the velocity of nanofluid because the electrical force introduces accelerating body force which acts in the direction of the electrical force. The Lorentz force accelerates the boundary layer flow. This force also increases the thickness of the momentum boundary layer. Hence, it results in a reduction in skin friction coefficient. Figure 3(c) presents the effect of magnetic parameter $M$ on velocity profile. From Figure 3(c), we conclude that a rise in magnetic parameter $M$ causes reduction in the velocity of fluid. An increase in magnetic parameter $M$ generates a resistive force called Lorentz force in the fluid layers. This resistive force causes the reduction in velocity profile. Figure $3(d)$ is constructed to know the effect of power index $n$ on velocity distribution. It is analyzed that for higher values of power index $n$, the velocity profile remains unchanged. Figure 4(a) reflects the influence $\theta_{r}$ has on the velocity. The larger values of viscosity parameter $\theta_{r}$ intensify the skin friction, which causes the reduction in the velocity profile. Figure 4(b) demonstrates the effect of fluid parameter $N$ on the velocity distribution. It can be seen that the velocity profile is enhanced for higher values of $N$. Figure 4(c) is constructed to know the effects of Grashof number $\mathrm{Gr}$ on velocity distribution. The velocity goes down with larger values of 


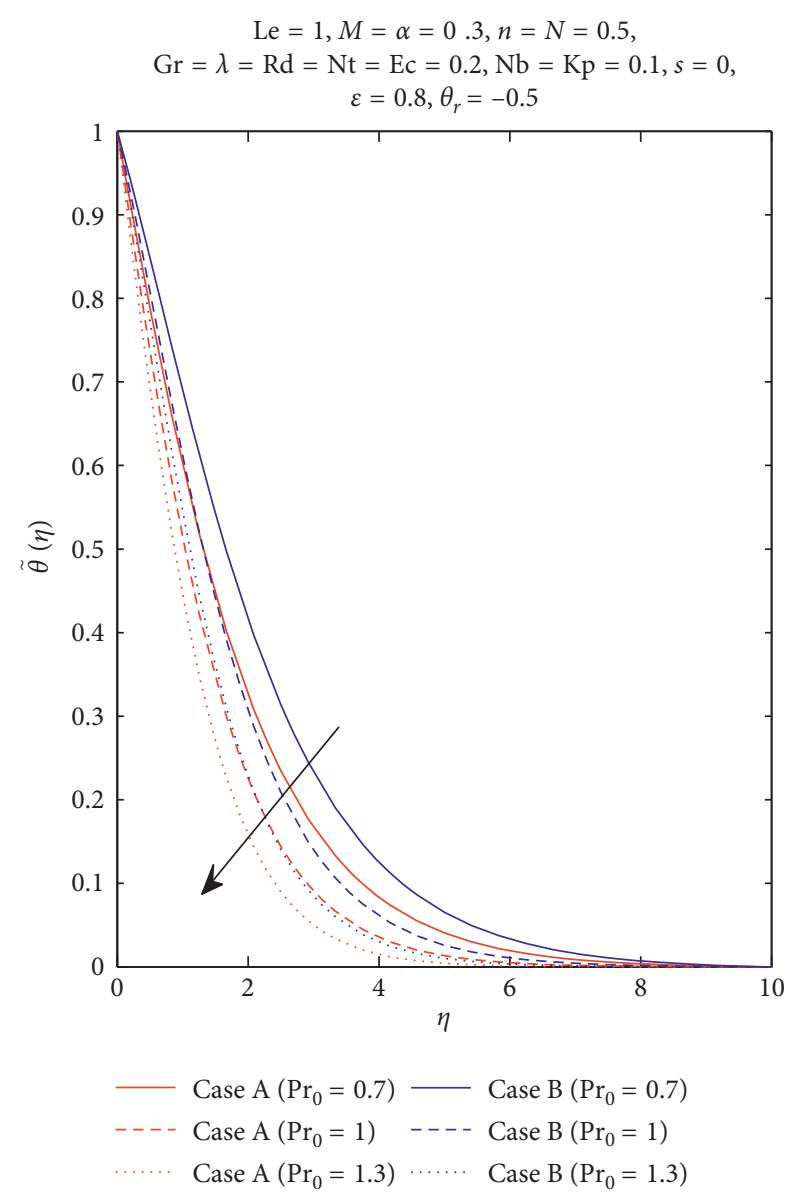

(a)

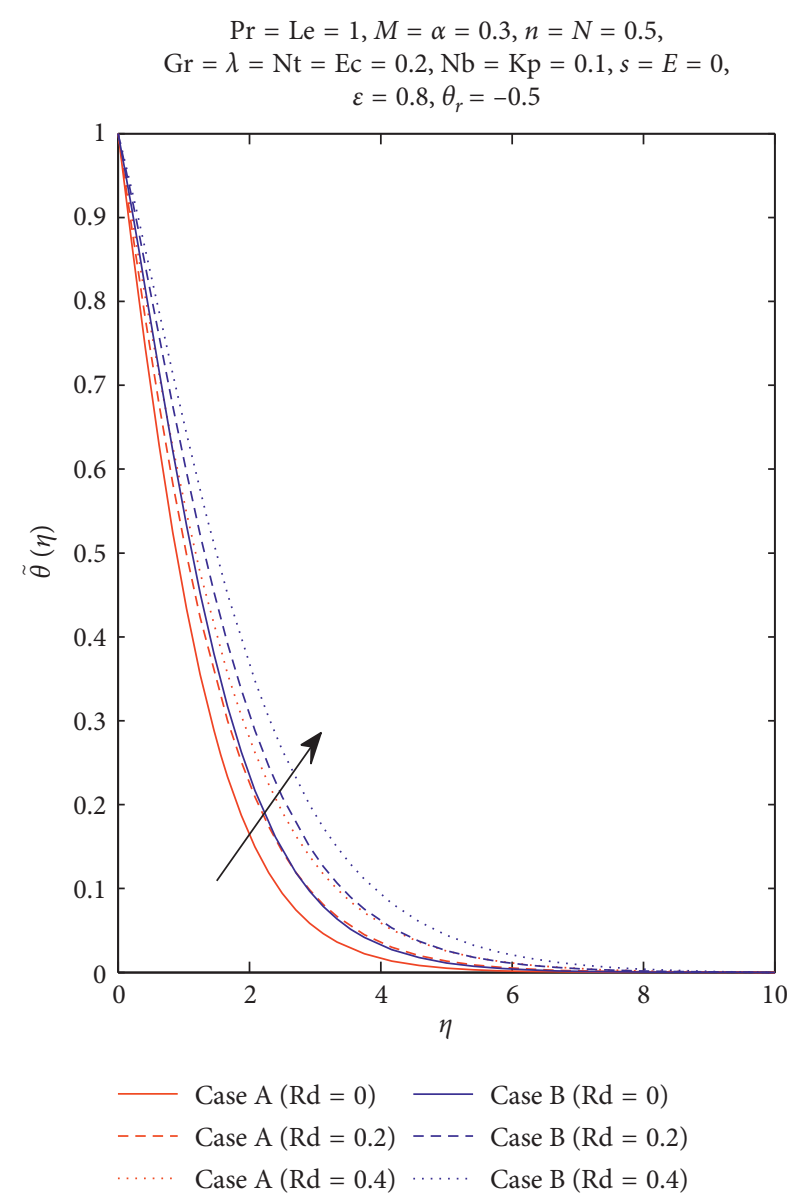

(b)

Figure 6: Continued. 


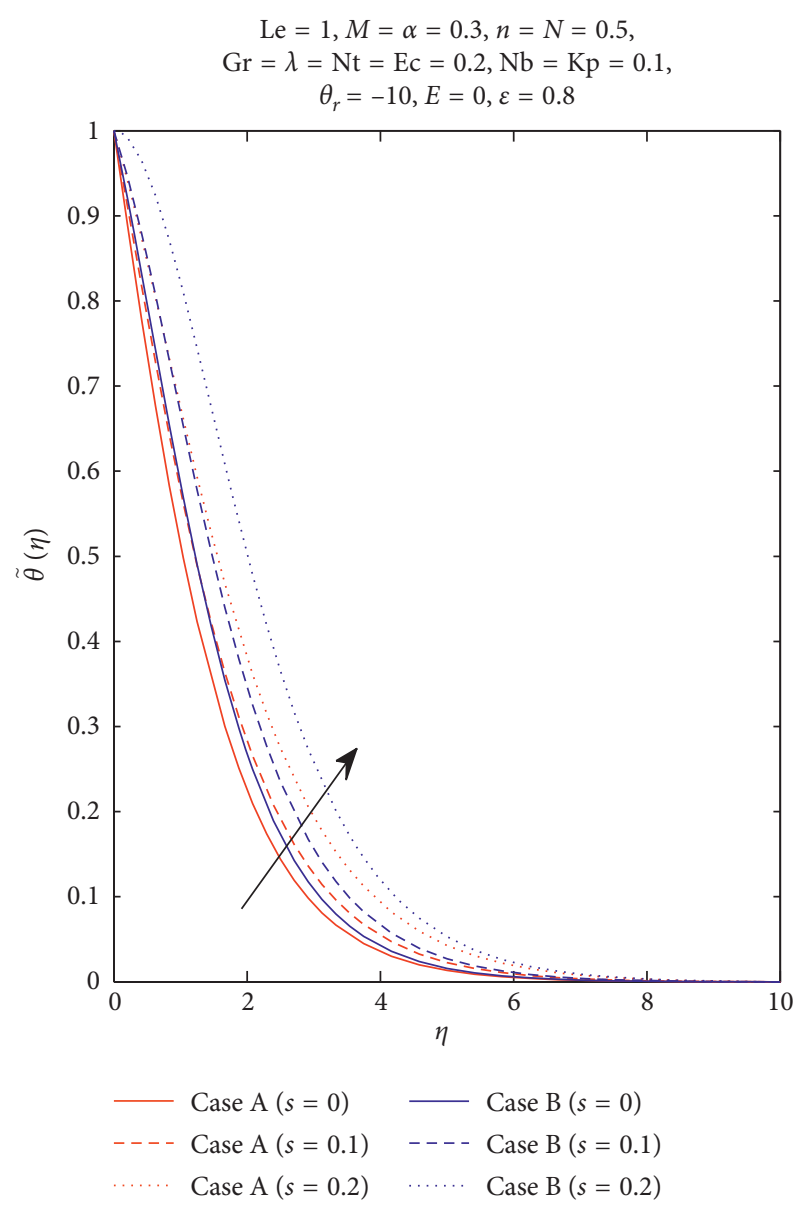

(c)

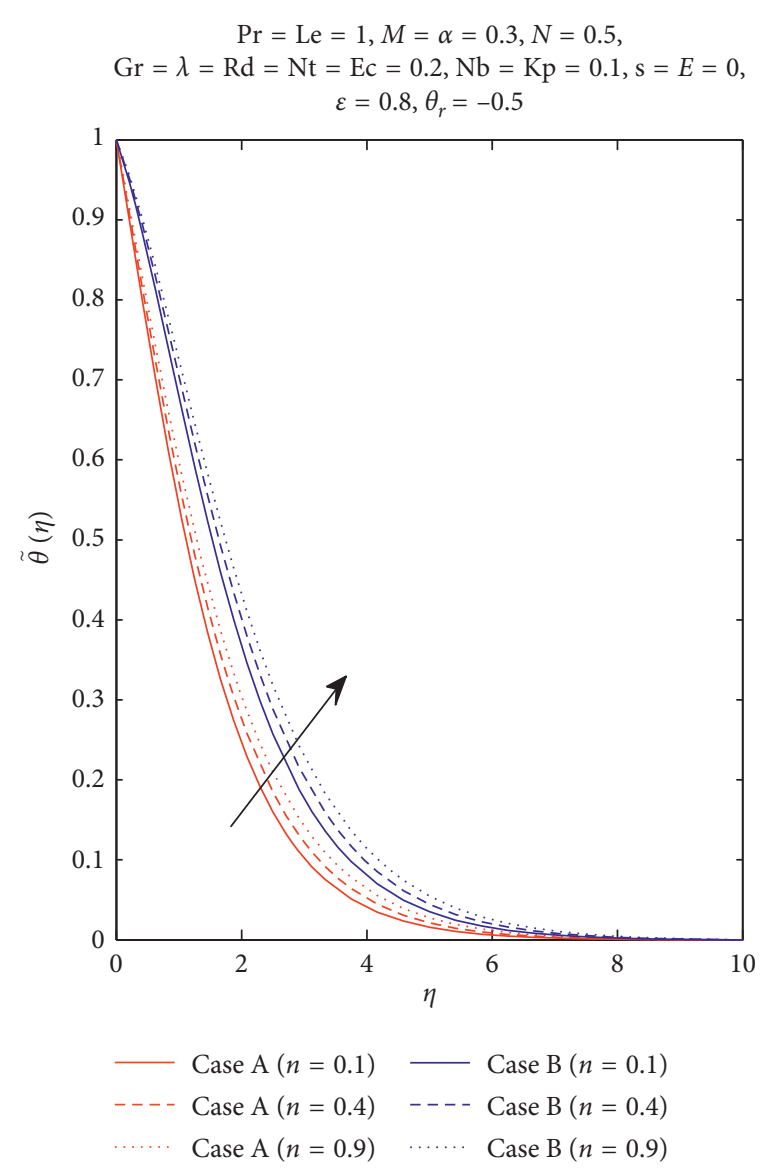

(d)

FIgURE 6: Temperature considerations supplied against (a) $\operatorname{Pr}_{0}$, (b) $\mathrm{Rd}$, (c) $s$, and (d) $n$.

Grashof number Gr. Figure 4(d) highlights the effect of porosity parameter $\mathrm{Kp}$ on velocity profiles. It is seen that velocity profile decreases with the increase in Kp. Figure 5(a) presents the effect of $\lambda$ on velocity profile. It is analyzed that there is no significant change in velocity profile for different values of $\lambda$ in both Cases $A$ and $B$. The thermal boundary layer thickness decreases for Case $B$ when compared with the associated results obtained for Case A. Figure 5(b) demonstrates the effect of variable thickness parameter $\alpha$ on temperature profile. It is observed that an enhancement in variable thickness parameter $\alpha$ reduces the temperature profile. The behavior of electric field parameter $E$ on temperature profile is portrayed in Figure 5(c). It is noted that enhancement in electric field parameter $E$ decreases the thermal boundary layer thickness. Figure $5(\mathrm{~d})$ is presented to describe the influence of small thermal conductivity parameter $\epsilon$ on temperature profile. It is examined that increment in small thermal conductivity $\epsilon$ causes enhancement in temperature profile. Figure 6(a) is presented to examine the effect of Prandtl number $\operatorname{Pr}_{0}$ on temperature profile. It is noticed that increasing parameter $\operatorname{Pr}_{0}$ reduces thermal conductivity of fluid which decreases temperature profile. Figure 6(b) illustrates the effect of radiation parameter Rd on temperature profile. It is seen that small increment in radiation parameter $\mathrm{Rd}$ intensifies the temperature profile because an increment in radiation parameter $\mathrm{Rd}$ provides more heat to fluid that causes enhancement in the thermal boundary layer thickness. Figure 6(c) illustrates the effect of heat source parameter $s$ on temperature profile. It is observed that by increasing heat source parameter $s$, the temperature of the fluid increases, and hence the thermal boundary layer increases. Figure $6(\mathrm{~d})$ is plotted to perceive the effect of power index parameter $n$ on temperature profile. It is noted that increasing power index parameter $n$ increases the temperature profile. Figures 7(a) and 7(b) show the effect of Brownian motion parameter $\mathrm{Nb}$ on the temperature and concentration profiles, respectively. It is noticed that for higher values of $\mathrm{Nb}$, there is no significant increase or decrease in temperature profile, but by increasing $\mathrm{Nb}$, the concentration boundary layer thickness increases. Figures 7(c) and 7(d) describe the effect of $\mathrm{Nt}$ on temperature and concentration profile, respectively. It is found that an increment in thermophoretic parameter $\mathrm{Nt}$ enhances both temperature and concentration profiles. Figure 8 depicts the influence of Le on concentration profile. We have seen that for larger values of Lewis number Le, the concentration boundary layer thickness is decreased. The reason is that increasing Lewis number Le reduces the 


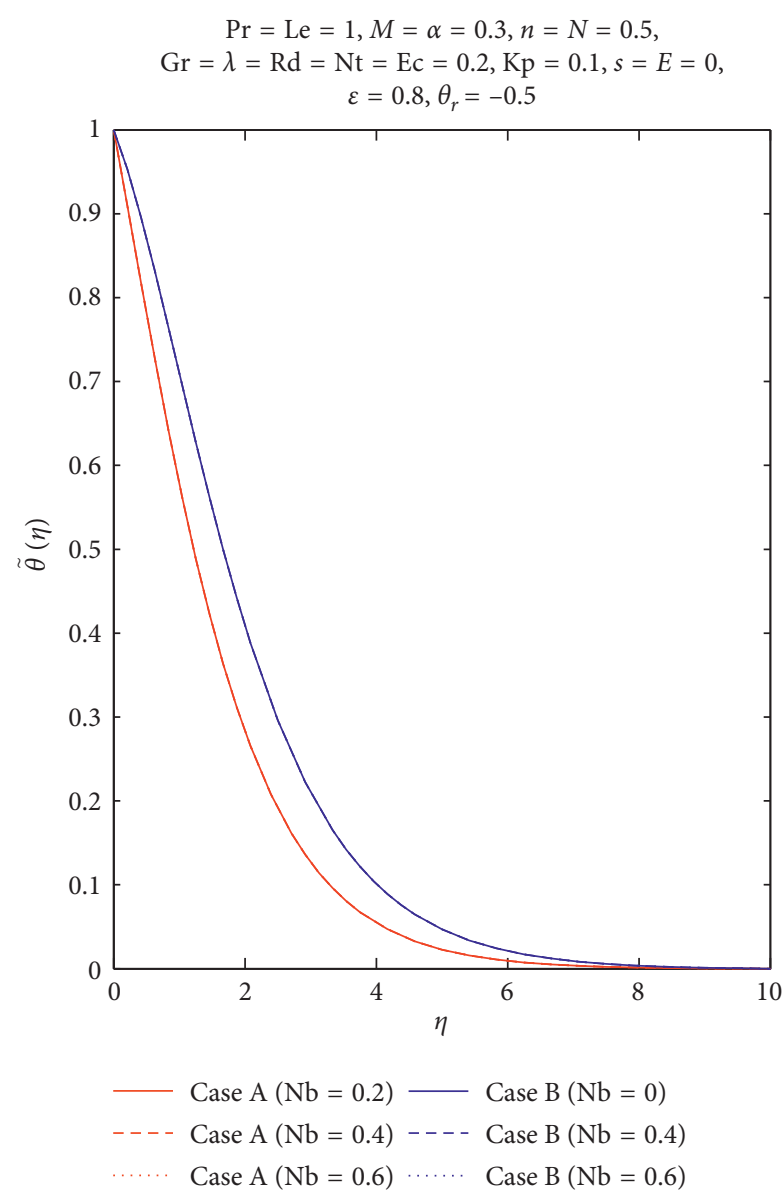

(a)

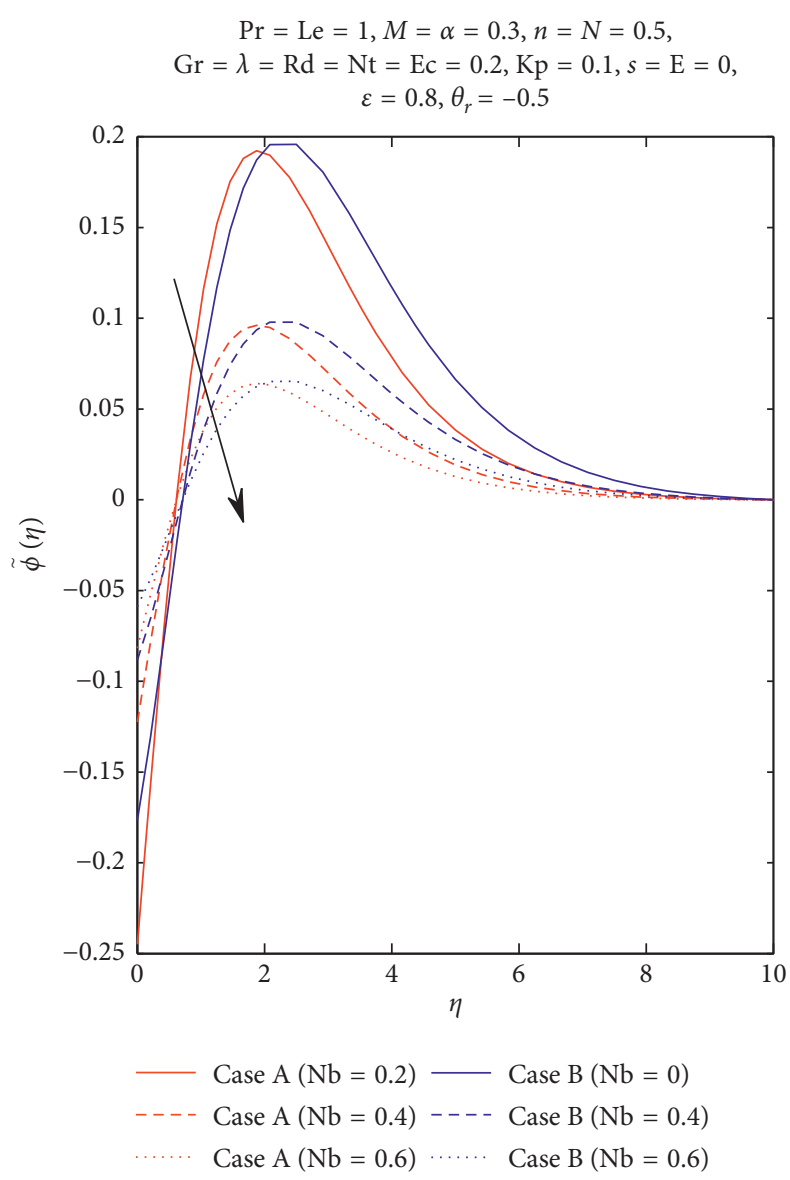

(b)

Figure 7: Continued. 


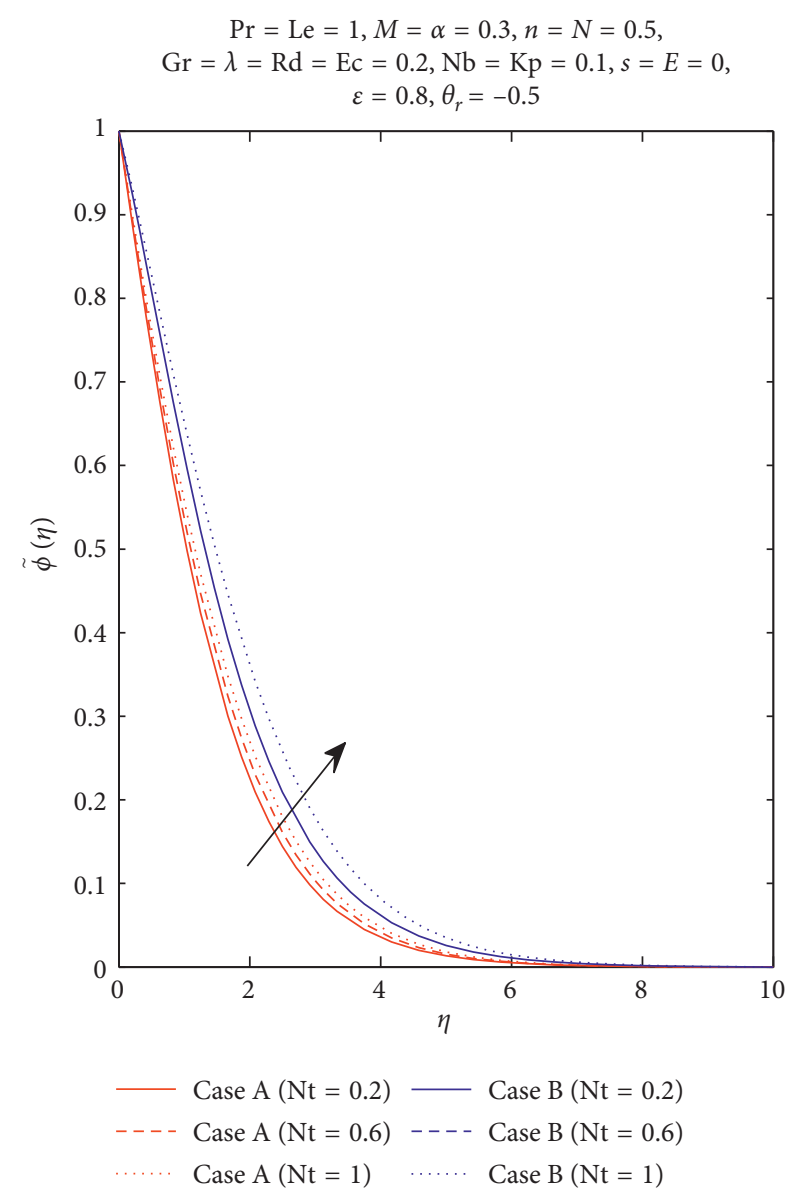

(c)

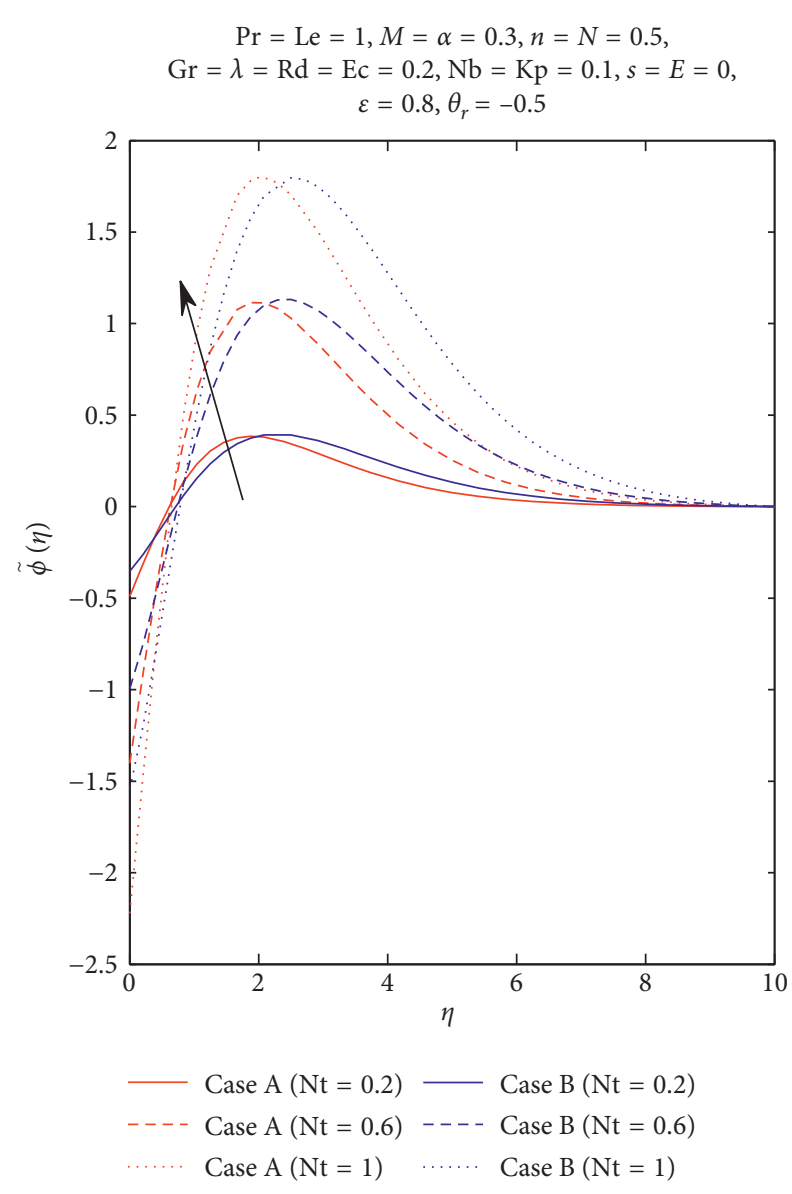

(d)

Figure 7: Temperature and concentration considerations for different $\mathrm{Nb}$ and $\mathrm{Nt}$.

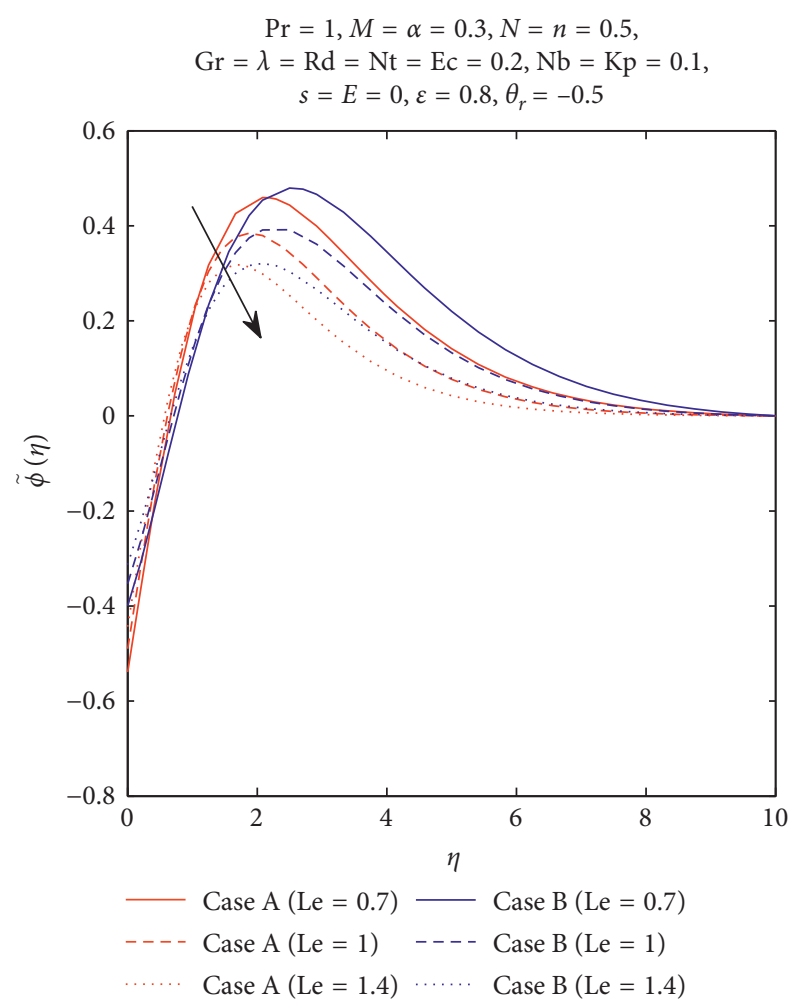

Figure 8: Concentration profile for different Le. 
concentration rate diffusivity, and hence the concentration profile decreases.

\section{Conclusions}

The present study gives the results of two-dimensional EMHD flow of Powell-Eyring nanofluid over a nonlinear stretching sheet in a porous medium with variable thickness and variable liquid properties. The numerical solutions are obtained with the help of SFDM for constant fluid properties. However, $b v p 4 c$ is implemented for variable fluid properties. The key findings of the present study are as follows:

(i) Momentum boundary layer thickness is higher for electric field $E$, parameter $N$, and Grashof number $\mathrm{Gr}$, whereas it is going down for magnetic parameter $M$, porosity parameter $K_{p}$, variable thickness parameter $\alpha$, and fluid viscosity parameter $\theta_{r}$ (for variable properties only).

(ii) Momentum boundary layer remains almost constant when we increase the power index parameter $n$ and fluid property parameter $\lambda$.

(iii) Thermal boundary layer thickness increases with the increase of radiation parameter $\mathrm{Rd}$, small thermal conductivity parameter $\epsilon$, heat source parameter $s$, power index $n$, and thermophoretic parameter Nt.

(iv) Thermal boundary layer thickness decreases for variable thickness parameter $\alpha$, electric field parameter $E$, and Prandtl number $\operatorname{Pr}_{0}$, whereas there is a constant effect in thermal boundary layer when increasing Brownian motion parameter $\mathrm{Nb}$.

(v) Concentration boundary layer thickness decreases with the increase of $\mathrm{Nt}$, whereas it increases with the increase of $\mathrm{Nb}$ and Le.

(vi) Difference of Cases A and B is seen with the help of graphs. Graphs show that boundary layer thickness of Case B is different when compared to Case A. Furthermore, the skin friction coefficient is higher for constant fluid properties when seen in comparison with variable properties. The same is observed for the local Nusselt number.

(vii) Lastly, the accuracy of the SFDM has been observed when comparison is drawn with the literature.

\footnotetext{
Abbreviation

$b$ : $\quad$ Positive constant

$a_{0}$ : $\quad$ Uniform velocity

$\left(u_{1}, v_{1}\right)$ : The velocity components $\left(\mathrm{ms}^{-1}\right)$

$\mu_{1}$ : The coefficient of viscosity (Pas)

$\rho_{1}: \quad$ The density of fluid $\left(\mathrm{kg} \mathrm{m}^{-3}\right)$

$E_{o}: \quad$ Uniform electric field factor

E: $\quad$ Electrical field parameter

$E_{1}^{*}: \quad$ Applied electric field

Kp: $\quad$ Permeability parameter
}

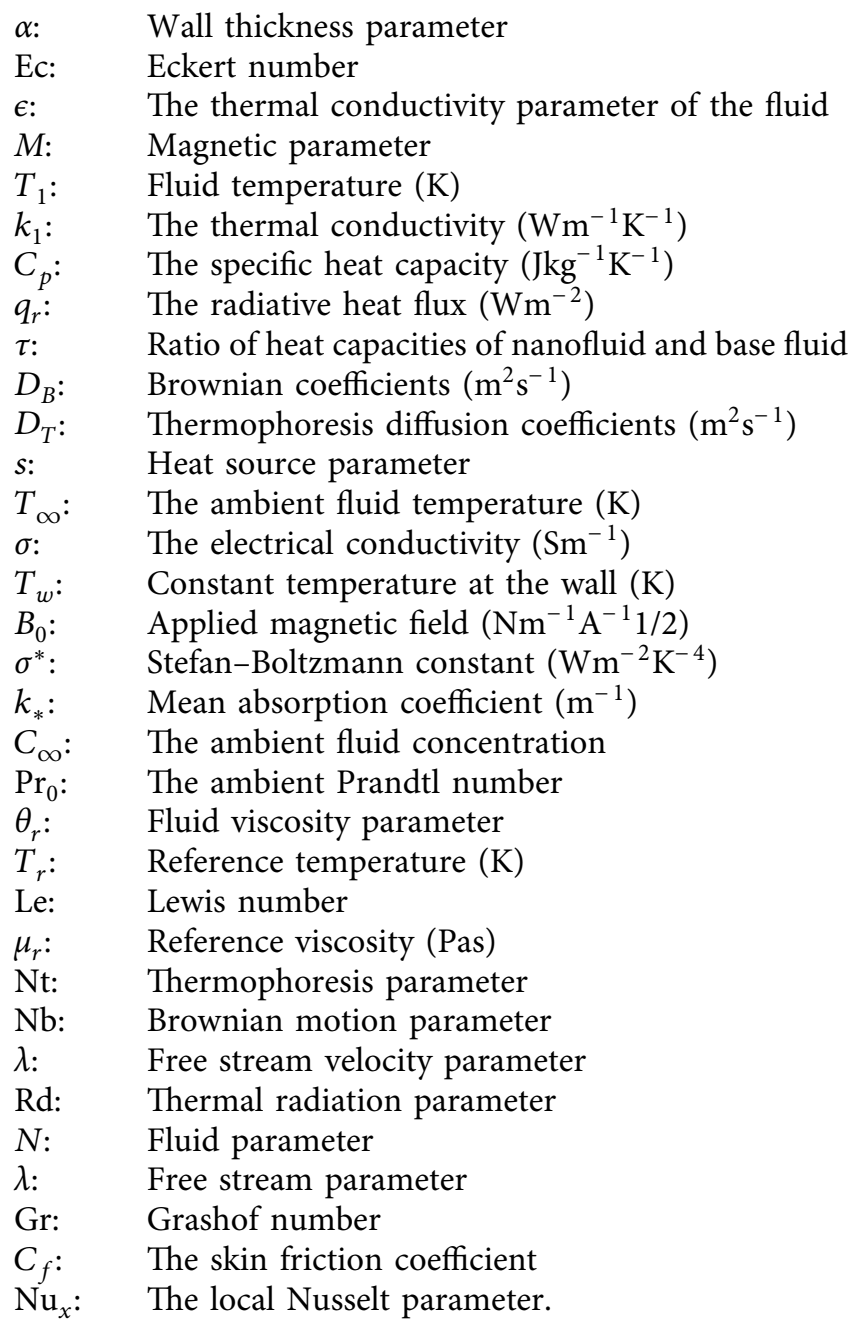

\section{Data Availability}

No experimental data were used to support this study.

\section{Conflicts of Interest}

The authors declare that there are no conflicts of interest.

\section{Authors' Contributions}

MI and MAF jointly proposed the research concept and then proceeded in developing the mathematical model. MAF wrote the introduction of the manuscript. MI, AM, ZHS, and TI contributed in the numerical part under the guidance of MAF. MI, MAF, and TI debated the detailed results of the numerical technique and the trend of different variables in fluid flow. MAF, AM, and ZHS discussed the structure of the paper.

\section{Acknowledgments}

AM would like to thank Mathematics Teaching and Learning, research group within the Department of Mathematics, Bodø, Nord University, for the partial support. ZHS would like to thank the Department of 
Mathematics, University of the Punjab, Lahore, for the partial support.

\section{References}

[1] Y. S. Daniel, Z. A. Aziz, Z. Ismail, and F. Salah, "Impact of thermal radiation on electrical MHD flow of nanofluid over nonlinear stretching sheet with variable thickness," Alexandria Engineering Journal, vol. 57, no. 3, pp. 2187-2197, 2018.

[2] T. Fang, J. Zhang, and Y. Zhong, "Boundary layer flow over a stretching sheet with variable thickness," Applied Mathematics and Computation, vol. 218, no. 13, pp. 7241-7252, 2012.

[3] S. M. Atif, S. Hussain, and M. Sagheer, "Magnetohydrodynamic stratified bioconvective flow of micropolar nanofluid due to gyrotactic microorganisms," AIP Advances, vol. 9, no. 2, Article ID 025208, 2019.

[4] A. Alsaedi, M. I. Khan, M. Farooq, N. Gull, and T. Hayat, "Magnetohydrodynamic (MHD) stratified bioconvective flow of nanofluid due to gyrotactic microorganisms," Advanced Powder Technology, vol. 28, no. 1, pp. 288-298, 2017.

[5] T. Hayat, M. I. Khan, M. Waqas, A. Alsaedi, and T. Yasmeen, "Diffusion of chemically reactive species in third grade fluid flow over an exponentially stretching sheet considering magnetic field effects," Chinese Journal of Chemical Engineering, vol. 25, no. 3, pp. 257-263, 2017.

[6] M. I. Khan, M. Z. Kiyani, M. Y. Malik, T. Yasmeen, M. W. A. Khan, and T. Abbas, "Numerical investigation of magnetohydrodynamic stagnation point flow with variable properties," Alexandria Engineering Journal, vol. 55, no. 3, pp. 2367-2373, 2016.

[7] T. Hayat, M. I. Khan, M. Waqas, A. Alsaedi, and M. Farooq, "Numerical simulation for melting heat transfer and radiation effects in stagnation point flow of carbon-water nanofluid," Computer Methods in Applied Mechanics and Engineering, vol. 315, pp. 1011-1024, 2017.

[8] T. Yasmeen, T. Hayat, M. I. Khan, M. Imtiaz, and A. Alsaedi, "Ferrofluid flow by a stretched surface in the presence of magnetic dipole and homogeneous-heterogeneous reactions," Journal of Molecular Liquids, vol. 223, pp. 1000-1005, 2016.

[9] T. Hayat, M. I. Khan, M. Waqas, T. Yasmeen, and A. Alsaedi, "Viscous dissipation effect in flow of magnetonanofluid with variable properties," Journal of Molecular Liquids, vol. 222, pp. 47-54, 2016.

[10] T. Hayat, M. I. Khan, A. Alsaedi, and M. I. Khan, "Homogeneous-heterogeneous reactions and melting heat transfer effects in the MHD flow by a stretching surface with variable thickness," Journal of Molecular Liquids, vol. 223, pp. 960968, 2016.

[11] T. Hayat, M. I. Khan, M. Farooq, N. Gull, and A. Alsaedi, "Unsteady three-dimensional mixed convection flow with variable viscosity and thermal conductivity," Journal of Molecular Liquids, vol. 223, pp. 1297-1310, 2016.

[12] K. Das, R. P. Sharma, and A. Sarkar, "Heat and mass transfer of a second grade magnetohydrodynamic fluid over a convectively heated stretching sheet," Journal of Computational Design and Engineering, vol. 3, no. 4, pp. 330-336, 2016.

[13] K. Bhattacharyya, M. S. Uddin, and G. C. Layek, "Exact solution for thermal boundary layer in Casson fluid flow over permeable shrinking sheet with variable wall temperature and thermal radiation," Alexandria Engineering Journal, vol. 55, no. 2, pp. 1703-1712, 2016.

[14] R. S. Raju, G. Jithender Reddy, J. A. Rao, and M. M. Rashidi, "Thermal diffusion and diffusion thermo effects on an unsteady heat and mass transfer magnetohydrodynamic natural convection Couette flow using FEM," Journal of Computational Design and Engineering, vol. 3, no. 4, pp. 349-362, 2016.

[15] M. Farooq, A. Anjum, T. Hayat, and A. Alsaedi, "Melting heat transfer in the flow over a variable thicked Riga plate with homogeneous-heterogeneous reactions," Journal of Molecular Liquids, vol. 224, pp. 1341-1347, 2016.

[16] A. Kumar, V. Sugunamma, N. Sandeep, and R. R. JV, "Impact of Brownian motion and thermophoresis on bioconvective flow of nanoliquids past a variable thickness surface with slip effects," Multidiscipline Modeling in Materials and Structures, vol. 15, no. 1, pp. 103-132, 2019.

[17] K. Anantha, B. Ramadevi, and V. Sugunamma, "Impact of Lorentz force on unsteady bio convective flow of Carreau fluid across a variable thickness sheet with non-Fourier heat flux model,"Defect and Diffusion Forum, vol. 387, pp. 474-497, Trans Tech Publications Ltd., Switzerland, 2018.

[18] K. A. Kumar, J. R. Reddy, V. Sugunamma, and N. Sandeep, "Magnetohydrodynamic Cattaneo-Christov flow past a cone and a wedge with variable heat source/sink," Alexandria Engineering Journal, vol. 57, no. 1, pp. 435-443, 2018.

[19] Y. S. Daniel, Z. A. Aziz, Z. Ismail, and F. Salah, "Effects of slip and convective conditions on MHD flow of nanofluid over a porous nonlinear stretching/shrinking sheet," Australian Journal of Mechanical Engineering, vol. 16, no. 3, pp. 213-229, 2018.

[20] T. Salahuddin, M. Y. Malik, A. Hussain, S. Bilal, and M. Awais, "MHD flow of Cattanneo-Christov heat flux model for Williamson fluid over a stretching sheet with variable thickness: using numerical approach," Journal of Magnetism and Magnetic Materials, vol. 401, pp. 991-997, 2016.

[21] S. Reddy, K. Naikoti, and M. M. Rashidi, "MHD flow and heat transfer characteristics of Williamson nanofluid over a stretching sheet with variable thickness and variable thermal conductivity," Transactions of A. Razmadze Mathematical Institute, vol. 171, no. 2, pp. 195-211, 2017.

[22] M. Y. Malik, M. Khan, T. Salahuddin, and I. Khan, "Variable viscosity and MHD flow in Casson fluid with CattaneoChristov heat flux model: using Keller box method," Engineering Science and Technology, an International Journal, vol. 19, no. 4, pp. 1985-1992, 2016.

[23] G. K. Ramesh, B. C. Prasannakumara, B. J. Gireesha, and M. M. Rashidi, "Casson fluid flow near the stagnation point over a stretching sheet with variable thickness and radiation," Journal of Applied Fluid Mechanics, vol. 9, no. 3, pp. 11151022, 2016.

[24] O. D. Makinde, F. Mabood, W. A. Khan, and M. S. Tshehla, "MHD flow of a variable viscosity nanofluid over a radially stretching convective surface with radiative heat," Journal of Molecular Liquids, vol. 219, pp. 624-630, 2016.

[25] K. V. Prasad, K. Vajravelu, H. Vaidya, and R. A. Van Gorder, "MHD flow and heat transfer in a nanofluid over a slender elastic sheet with variable thickness," Results in Physics, vol. 7, pp. 1462-1474, 2017.

[26] M. Turkyilmazoglu, "Thermal radiation effects on the timedependent MHD permeable flow having variable viscosity," International Journal of Thermal Sciences, vol. 50, no. 1, pp. 88-96, 2011.

[27] K. V. Prasad, A. Sujatha, K. Vajravelu, and I. Pop, "MHD flow and heat transfer of a UCM fluid over a stretching surface with variable thermophysical properties," Meccanica, vol. 47, no. 6, pp. 1425-1439, 2012.

[28] K. Vajravelu, K. V. Prasad, and C.-O. Ng, "Unsteady convective boundary layer flow of a viscous fluid at a vertical 
surface with variable fluid properties," Nonlinear Analysis: Real World Applications, vol. 14, no. 1, pp. 455-464, 2013.

[29] K. Das, "Impact of thermal radiation on MHD slip flow over a flat plate with variable fluid properties," Heat and Mass Transfer, vol. 48, no. 5, pp. 767-778, 2012.

[30] S. Mukhopadhyay, "Effects of thermal radiation and variable fluid viscosity on stagnation point flow past a porous stretching sheet," Meccanica, vol. 48, no. 7, pp. 1717-1730, 2013.

[31] M. M. Rahman and I. A. Eltayeb, "Convective slip flow of rarefied fluids over a wedge with thermal jump and variable transport properties," International Journal of Thermal Sciences, vol. 50, no. 4, pp. 468-479, 2011.

[32] T. Hayat, M. Farooq, A. Alsaedi, and Z. Iqbal, "Melting heat transfer in the stagnation point flow of Powell-Eyring fluid," Journal of Thermophysics and Heat Transfer, vol. 27, no. 4, pp. 761-766, 2013.

[33] M. Jalil and S. Asghar, "Flow and heat transfer of PowellEyring fluid over a stretching surface: a lie group analysis," Journal of Fluids Engineering, vol. 135, no. 12, 2013.

[34] A. Mushtaq, M. Mustafa, T. Hayat, M. Rahi, and A. Alsaedi, "Exponentially stretching sheet in a Powell-Eyring Fluid: numerical and series solutions," Zeitschrift für Naturforschung A, vol. 68, no. 12, pp. 791-798, 2013.

[35] T. Javed, N. Ali, Z. Abbas, and M. Sajid, "Flow of an EyringPowell non-Newtonian fluid over a stretching sheet," Chemical Engineering Communications, vol. 200, no. 3, pp. 327-336, 2013.

[36] M. Mustafa, M. Nawaz, T. Hayat, and A. Alsaedi, "MHD boundary layer flow of second-grade nanofluid over a stretching sheet with convective boundary conditions," Journal of Aerospace Engineering, vol. 27, no. 4, Article ID 04014006, 2014.

[37] T. G. Motsumi and O. D. Makinde, "Effects of thermal radiation and viscous dissipation on boundary layer flow of nanofluids over a permeable moving flat plate," Physica Scripta, vol. 86, no. 4, Article ID 045003, 2012.

[38] F. M. White, Fluid Mechanics, McGraw-Hill, New York, NY, USA, 2003.

[39] H. I. Andersson and J. B. Aarseth, "Sakiadis flow with variable fluid properties revisited," International Journal of Engineering Science, vol. 45, no. 2-8, pp. 554-561, 2007.

[40] K. U. Rehman, N. U. Saba, M. Y. Malik, and I. Zehra, "Nanoparticles individualities in both Newtonian and Casson fluid models by way of stratified media: a numerical analysis," The European Physical Journal E, vol. 41, no. 3, pp. 1-10, 2018.

[41] U. Ali, K. U. Rehman, and M. Y. Malik, "The influence of MHD and heat generation/absorption in a Newtonian flow field manifested with a Cattaneo-Christov heat flux model," Physica Scripta, vol. 94, no. 8, Article ID 085217, 2019.

[42] K. U. Rehman, M. Y. Malik, I. Zehra, and M. S. Alqarni, "Group theoretical analysis for MHD flow fields: a numerical result," Journal of the Brazilian Society of Mechanical Sciences and Engineering, vol. 41, no. 3, p. 156, 2019.

[43] M. Y. Malik, "Application of shooting method on MHD thermally stratified mixed convection flow of non-Newtonian fluid over an inclined stretching cylinder," Journal of Physics: Conference Series, vol. 822, no. 1, Article ID 012012, 2017.

[44] K. U. Rehman, Q. M. Al-Mdallal, and M. Y. Malik, "Symmetry analysis on thermally magnetized fluid flow regime with heat source/sink," Case Studies in Thermal Engineering, vol. 14, Article ID 100452, 2019.

[45] M. Irfan, M. A. Farooq, and T. Iqra, "A new computational technique design for EMHD nanofluid flow over a variable thickness surface with variable liquid characteristics," Frontiers in Physics, vol. 8, pp. 1-14, 2020.

[46] T. Y. Na, Ed., Computational Methods in Engineering Boundary Value Problems, Academic Press, Cambridge, MA, USA, 1980.

[47] L. Thomas, Elliptic Problems in Linear Differential Equations over a Network: Watson scientific Computing Laboratory, Columbia University, New York, NY, USA, 1949.

[48] J. Kierzenka and L. F. Shampine, "A BVP solver based on residual control and the Maltab PSE," ACM Transactions on Mathematical Software, vol. 27, no. 3, pp. 299-316, 2001. 